\title{
Effet d'une réduction simultanée de la fertilisation azotée et du chargement sur les performances des vaches laitières et la valorisation du pâturage
}

\author{
Luc Delaby $^{a^{*}}$, Jean-Louis Peyraud ${ }^{\mathrm{a}}$ \\ avec la collaboration technique d'Alain Bouttier ${ }^{\mathrm{b}}$, \\ Jean-Rémi Peccatte ${ }^{b}$ \\ ${ }^{a}$ Station de recherches sur la vache laitière, Inra, 35590 Saint-Gilles, France \\ 'Inra, domaine expérimental du Pin au Haras, 61310 Exmes, France
}

(Reçu le 14 février 1997 ; accepté le 10 septembre 1997)

\begin{abstract}
Effect of a simultaneous reduction of nitrogen fertilization and stocking rate on grazing dairy cow performances and pasture utilization. Three levels of nitrogen fertilization and stocking rate corrected to obtain the same level of herbage allowance were applied to permanent and grazing meadows in Normandy over 5 years: high $=320 \mathrm{~kg} \mathrm{~N} / \mathrm{ha} /$ year and $2.5 \mathrm{cows} / \mathrm{ha}$; medium $=100$ and 2.0 ; low $=0$ and 1.7. Each year, 18 dairy cows were assigned to each treatment for the whole grazing season. Weather conditions in the first 2 years only permitted 90 days of grazing in the spring. Three full grazing seasons ( 190 days) followed. Reducing nitrogen fertilization systematically reduced the biomass available per hectare and per cycle $(2540,2230$ and $1760 \mathrm{~kg} \mathrm{DM}$ for the $\mathrm{H}, \mathrm{M}$ and $\mathrm{L}$ levels, respectively), the CP content (225, 177 and $158 \mathrm{~g} / \mathrm{kg} \mathrm{DM}$, respectively) and the digestibility of the grass available $(0.74,0.71$ and 0.70 units, respectively). In spite of the total suppression of nitrogen fertilization, the annual productivity of the meadow reached 8.5 DM under low treatment and did not decrease throughout the 5 years of the experiment. In spite of a small decrease in the nutritive value of the grass and with a same mean of herbage allowance, the average individual milk yield, the butter fat and protein contents of the five springs and of the last 3 years $(27.3$ and $22.1 \mathrm{~kg} /$ day, respectively), were not different under all three treatments. There were inter-annual differences in treatment response, especially in the spring, associated to wider variations of available grass under the low treatment. Supplementation of $100 \mathrm{~kg} \mathrm{~N} / \mathrm{ha} /$ year in the medium protocol reduced that inter-annual variability. Milk yield per hectare decreased in the same proportions as the stocking rate and on average amounted to $15.6 \mathrm{~kg}$ milk/ha less for each $\mathrm{kg}$ of nitrogen suppressed from 320 to $100 \mathrm{~kg} \mathrm{~N} / \mathrm{ha} /$ year and to 19.0 $\mathrm{kg} \mathrm{milk} / \mathrm{ha}$ for each $\mathrm{kg}$ of nitrogen suppressed from 100 to $0 \mathrm{~kg} \mathrm{~N} / \mathrm{ha} /$ year. These results are to be
\end{abstract}

* Correspondance et tirés à part. 
corrected for local agro-climatic potential because their dependence on soil nitrogen supply increases as nitrogen fertilization is reduced. (@ Elsevier/Inra.)

grazing / dairy cow / nitrogen fertilization / stocking rate / grass chemical composition

\begin{abstract}
Résumé - Trois niveaux de fertilisation azotée et de chargement adaptés afin d'offrir la même quantité d'herbe par vache (Haut : $320 \mathrm{~kg} \mathrm{~N} / \mathrm{ha} /$ an et 2,5 vaches/ha ; Moyen : 100 et 2,0; Bas : 0 et 1,7$)$ ont été appliqués durant 5 années consécutives sur des prairies permanentes et temporaires en Normandie (Inra Le Pin au Haras). Chaque année, 18 vaches laitières ont été affectées à chaque traitement pour toute la saison de pâturage. Lors des 2 premières années, les conditions climatiques n'ont permis que $90 \mathrm{j}$ de pâturage au printemps. Ensuite, 3 saisons complètes de pâturage (190 j) ont été réalisées. La réduction de la fertilisation azotée a entraîné systématiquement une réduction de la biomasse offerte par hectare et par cycle $(2540,2230$ et $1760 \mathrm{~kg}$ MS pour $\mathrm{H}, \mathrm{M}$ et $\mathrm{B})$, de la teneur en MAT $(225,177$ et $158 \mathrm{~g} / \mathrm{kg}$ MS respectivement) et de la digestibilité de l'herbe offerte $(0,74,0,71$ et 0,70 unité respectivement). Malgré la suppression de toute fertilisation azotée, la productivité annuelle de la prairie permanente du traitement Bas a atteint 8,5 t MS et n'a pas montré d'évolution défavorable au cours des 5 années. En moyenne, au cours des 5 printemps, comme des 3 dernières années, malgré la diminution de la valeur nutritive de l'herbe offerte, et grâce à des quantités d'herbe offertes et ingérées voisines, la production laitière individuelle (27,3 et $22,1 \mathrm{~kg} / \mathrm{j}$ respectivement) et les taux butyreux et protéique n'ont pas différé entre les traitements. Des différences de réponse aux traitements existent entre années, notamment au printemps, et sont associées aux variations de la disponibilité de l'herbe plus importantes sur le traitement Bas. L'apport de $100 \mathrm{~kg} \mathrm{~N} / \mathrm{ha} / \mathrm{an}$ sur le traitement Moyen a atténué cette variabilité inter-années. La productivité par hectare a été réduite dans les mêmes proportions que le chargement et correspond en moyenne à une diminution de $15,6 \mathrm{~kg}$ de lait/ha par $\mathrm{kg}$ d'azote épandu en moins entre 320 et $100 \mathrm{~kg} \mathrm{~N}$ et de $19,0 \mathrm{~kg}$ de lait/ha entre 100 et $0 \mathrm{~kg} \mathrm{~N} / \mathrm{ha} / \mathrm{an}$. Ces résultats devront être adaptés aux potentialités agroclimatiques locales car leur dépendance vis à vis de la fourniture d'azote par le sol est accrue avec la réduction de la fertilisation azotée.

(C) Elsevier / Inra.)
\end{abstract}

pâturage / vache laitière / fertilisation azotée / chargement / composition chimique de l'herbe

\section{INTRODUCTION}

En Europe de l'Ouest, au cours des années 1960-85, l'utilisation accrue de la fertilisation azotée combinée à un chargement adapté, mais toujours croissant [ 3 , 30], ont permis d'augmenter les performances par hectare de pâturage tout en maintenant les performances individuelles élevées [4, 28]. Dans ces conditions, le revenu des exploitations est alors étroitement lié au chargement et les volumes de lait produits par vache ou livrés par exploitation ne cessent de croître [36].

Depuis l'instauration des quotas laitiers, la réduction importante et conjointe du nombre d'exploitations et de vaches laitières a entraîné une libération continue de surfaces agricoles, notamment en zones herbagères. Simultanément à cette évolution des structures, des contraintes environnementales de plus en plus pressantes apparaissent dans les bassins laitiers intensifs. La fertilisation azotée élevée, sous forme organique ou minérale, est désormais considérée comme un facteur qui accroît les risques de pollution des eaux par les nitrates [19,21], même sous prairies pâturées [49]. La maximisation des performances par hectare ne constitue donc plus l'unique objectif des systèmes herbagers. Il importe maintenant de préciser les conditions de désintensification des prairies, d'étudier la pérennité de ces systèmes de pâturage et d'en analyser les conséquences sur les performances individuelles tes vaches laitières. 
Les effets de la fertilisation azotée sur la production, la structure du couvert végétal $[35,44]$ et la composition chimique de l'herbe $[15,53]$ ont été largement décrits à la lumière d'expériences réalisées sur prairies fauchées. Plus récemment, Peyraud et Astigarraga [42], ont analysé lors d'une revue bibliographique exhaustive, les conséquences spécifiques sur l'ingestion et la digestion d'une réduction de la fertilisation azotée lors d'une valorisation de l'herbe par le pâturage. La réduction de la fertilisation $\mathrm{N}$ pourrait entraîner des variations d'apports nutritifs préjudiciables à la production laitière, ce malgré l'élargissement des surfaces offertes aux ani$\operatorname{maux}[10,13]$.

À partir d'une expérience pluri-annuelle (5 ans) réalisée au Pin au Haras (département de l'Orne en Normandie), l'objectif de cet article est de préciser, à l'échelle d'une saison complète de pâturage (6 mois), les effets d'une réduction simultanée de la fertilisation azotée et du chargement sur la production et la qualité de l'herbe d'une part et les performances zootechniques d'autre part. Des résultats partiels correspondant aux 2 premières années, limitées aux trois cycles de pâturage de printemps $[9,39]$ ou aux 3 dernières années expérimentales [11] ont été présentés par ailleurs. Les conséquences de cette désintensification de la surface pâturée sur les restitutions d'azote par vache et par hectare de prairie ont fait l'objet d'une publication particulière [12], intégrée dans une description quantifiée du cycle de l'azote sous prairies pâturées.

\section{MATERIEL ET METHODES}

\subsection{Principes et traitements expérimentaux}

Trois niveaux de fertilisation azotée annuels ont été comparés au cours de 5 années successives (1990-94). Afin d'offrir la même quantité d'herbe aux animaux des trois traitements, la baisse de productivité des prairies moins fertilisées a été compensée par une réduction du chargement. Les surfaces utilisées étaient constituées de prairies permanentes pour la surface de base et de prairies temporaires pour les surfaces additionnelles.

Les trois niveaux de fertilisation ont été : Haut : $320 \mathrm{~kg}$ (respectivement 80606060 $60 \mathrm{~kg} \mathrm{~N} /$ cycle), Moyen : $100 \mathrm{~kg}$ (40 202020 0 ) et Bas : $0 \mathrm{~kg} \mathrm{~N} / \mathrm{ha}$ sur la surface toujours pâturée de prairies permanentes. Sur prairies temporaires, les niveaux $\mathrm{H}, \mathrm{M}$ et $\mathrm{B}$ ont été respectivement de $200(80404040), 40$ (40 000 ) et $0 \mathrm{~kg} \mathrm{~N} / \mathrm{ha}$. Les surfaces affectées à chaque traitement ont été définies à partir des travaux sur la productivité des prairies conduites à différents niveaux de fertilisation azotée réalisés durant 12 années par R. Laissus et D. Leconte (comm pers) sur le même site expérimental. Le chargement prévisible a atteint au printemps sur prairies permanentes (et en automne sur la surface totale) pour les traitements Haut, Moyen et Bas respectivement 5,0 (2,5), 3,8 $(2,0)$ et $3,1(1,7)$ vaches/ha. Les prairies des trois traitements ont été valorisées à même âge de repousses lors des différents cycles de pâturage.

\subsection{Prairies et conduite du pâturage}

Les prairies permanentes drainées $(14,4 \mathrm{ha})$ sont situées sur sols acides ( $\mathrm{pH}: 5,5$ à 6,0$)$, limono-argileux et riches en matière organique (MO : 9 à $10 \%$ ). Elles sont composées essentiellement de ray-grass anglais $(20 \%)$, pâturin commun et agrostis ( $15 \%$ ), houlque laineuse, vulpin et fléole $(10 \%)$, trèfle blanc (5 à $10 \%)$. Les prairies temporaires $(12,9$ ha - 5 à $6 \%$ de MO), semées au printemps 1991 (10/04), sont constituées d'une association de ray-grass anglais ( $c v$ Magella) et de trèfle blanc (cv Menna).

Tous les 2 ans, l'ensemble des prairies a reçu une fumure de fond de $160 \mathrm{~kg}$ de $P$, sans apports de $\mathrm{K}$ compte tenu des teneurs en potassium du sol et de la plante [47]. La fertilisation azotée prévue a été épandue, sous forme d'ammonitrate $(33,5 \% \mathrm{~N})$, entre le 15 et 25 mars pour le premier apport, puis après chaque sortie des animaux de la parcelle.

Le système de pâturage tournant simplifié [26] à trois parcelles au printemps et six parcelles au maximum en automne a été adopté. Les surfaces de prairies permanentes (trois 
blocs homogènes divisés chacun en 3 parcelles de respectivement 1,$20 ; 1,56$ et 1,92 ha pour les traitements $\mathrm{H}, \mathrm{M}$ et $\mathrm{B}$ ) ont été utilisées au pâturage principalement. Les surfaces de prairies temporaires ( 3 parcelles identiques de 1,$20 ; 1,44$ et 1,68 ha respectivement pour $\mathrm{H}, \mathrm{M}$ et B) ont été récoltées en ensilage au premier cycle et ont ensuite été pâturées à partir du mois de Juin. Les excédents d'herbe éventuels après cette date ont été récoltés sous forme de foin ou de balles rondes enrubannées.

Les 3 traitements ont été conduits de façon indépendante durant chaque cycle. Intra-traitement, la sortie de parcelle des animaux a été décidée lorsque la production laitière moyenne du lot au cours des 3 derniers jours a atteint $90 \%$ du maximum observé sur la parcelle [28]. Cependant, afin de débuter les cycles à même date pour les 3 traitements, la surface pâturée de la dernière parcelle d'un cycle a pu être réduite et l'excédent fauché. Une fauche de refus annuelle a généralement été réalisée après le deuxième ou le troisième passage des animaux sur toutes les parcelles de prairies permanentes.

\subsection{Animaux et complémentation}

Chaque année, 54 vaches laitières, dont $37 \%$ de primipares, de race Holstein et Normande $(22 \%$ de vaches normandes en moyenne) ont été réparties en trois lots équilibrés sur la base de leurs performances observées pendant la période de mise à l'herbe (14 j, début avril) et affectées à un traitement pour les 6 mois de l'expérience. Durant ces 2 semaines, les animaux d'un poids moyen de $642( \pm 54) \mathrm{kg}$, ont produit $32,6( \pm 7,1) \mathrm{kg}$ de lait à $42,3( \pm 5,2) \mathrm{g} / \mathrm{kg}$ de TB et $29,2( \pm 2,4) \mathrm{g} / \mathrm{kg}$ de TP pour un stade de lactation de $91( \pm 24) \mathrm{j}$. La mise à l'herbe s'est déroulée sur la première parcelle de chaque traitement.

La complémentation individuelle a alors été fixée à raison de $1 \mathrm{~kg}$ de concentré pour $3 \mathrm{~kg}$ de lait au dessus de $25 \mathrm{~kg}$ ( $22 \mathrm{~kg}$ pour les primipares) avec un apport maximum de $5 \mathrm{~kg} / \mathrm{j}$. Les quantités distribuées à chaque vache sont restées constantes durant toute l'expérience comme l'ont suggéré Hoden et al. [27]. Le niveau moyen d'apport de concentré, identique pour les 3 lots intra année, a été de $2,7( \pm 1,7)$ $\mathrm{kg} / \mathrm{j} ; 13,5 \%$ des animaux n'en ont pas reçu. Le concentré, $(1,10$ UFL et $277 \mathrm{~g}$ de MAT par $\mathrm{kg}$ MS) était composé de $54 \%$ de tourteaux de soja et colza tannés, $18 \%$ de pulpes de bette- raves, $22, ; \%$ de blé, $3 \%$ de mélasse et $2,5 \%$ de minéri ux. Les animaux ont reçu en plus $500 \mathrm{~g} / \mathrm{j}$ de CMV, comportant $50 \%$ de minéral $12 \mathrm{P} / 16 \mathrm{Ca} / 5 \mathrm{Mg}$. La distribution du concentré et du CMV a été réalisée individuellement à l'aide de distributeurs automatiques de concentré (DAC - Système compact d'alimentation C16 - Westfalia) accessibles aux animaux durant $1 \mathrm{~h}$ à $1 \mathrm{~h} 30$ après chacune des deux traites journalières.

\subsection{Mesures}

\subsubsection{Prairies}

À chaque entrée de parcelle, la biomasse présente a été mesurée par coupe à la motofaucheuse (six bandes de $10 \times 0,5 \mathrm{~m}$ réparties au hasard). Sur chaque bande, deux ou trois poignées d'herbe ont été prélevées et regroupées par traitement pour constituer un échantillon moyen par parcelle. À partir de 1992 , sur les prairies temporaires, un second échantillon a été prélevé afin de déterminer par tri et pesée séparée, la contribution pondérale du ray grass et du trèfle blanc à la biomase de la parcelle. Toute la biomasse verte fauchée a ensuite été récoltée et pesée par bande. La longueur exacte de chaque bande a été mesurée.

Afin de calculer la densité de l'herbe fauchée (DE en $\mathrm{kg} \mathrm{MS} / \mathrm{ha} / \mathrm{cm}$ récolté), la hauteur de l'herbe a été mesurée sur chaque bande (dix mesures) avant et après la fauche à l'aide d'un herbomètre à plateau $\left(30 \times 30 \mathrm{~cm}-4,5 \mathrm{~kg} / \mathrm{m}^{2}\right)$ automatisé [50].

La hauteur de l'herbe de l'ensemble de chaque parcelle avant l'entrée (HE en $\mathrm{cm}$ ) et à la sortie (HS en $\mathrm{cm}$ ) des vaches ou après une fauche de refus a également été mesurée avec l'herbomètre à plateau à raison de 150 mesures par hectare.

Lors des récoltes d'excédents d'herbe, la surface exacte fauchée a été déterminée et la totalité du fourrage récolté a été pesée.

La pluviométrie et les températures minimales et maximales quotidiennes ont été relevées à partir d'une station météorologique locale affiliée au réseau national et implantée à proximité des parcelles expérimentales.

\subsubsection{Animaux}

La production laitière individuelle a été mesurée tous les jours à l'aide de compteurs à 
lait débimétriques (Westfalia) lors des deux traites débutant à $6 \mathrm{~h} 30$ et $16 \mathrm{~h} 00$ environ. Les taux butyreux et protéique ont été déterminés par analyseur infrarouge (Milkoscan, Foss Electric, DK-3400 Hillerod, Danemark) à partir de six échantillons individuels hebdomadaires prélevés lors de six traites consécutives (LiLaNo - 50008 Saint-Lô).

À partir de 1991, la teneur en urée du lait [37] a été déterminée deux fois par cycle, en début et fin d'une même parcelle de prairies permanentes, sur un échantillon de lait de mélange par traitement prélevé lors de la traite du matin.

Les animaux ont été pesés après la traite du matin, soit une à deux fois à chaque cycle de pâturage, quelques jours après l'entrée sur la parcelle en 1990 et 91, soit une fois par semaine lors des 3 dernières années d'expérience. L'état d'engraissement des animaux a été noté par maniement [1] chaque année à trois reprises en avril, juillet et octobre par deux personnes.

Lors de chaque passage sur la deuxième parcelle de prairies permanentes et sur une parcelle de prairies temporaires, un échantillon de chaque bouse émise au champ en $24 \mathrm{~h}$ par les 18 vaches de chaque lot a été collecté à partir du troisième jour afin d'estimer la digestibilité moyenne de la matière organique (MO) du régime (dMOr) consommé [39]

Les quantités quotidiennes de concentré ingérées ont été calculées pour chaque vache par différence entre le nombre de doses programmé et le nombre de doses non consommé fourni par les enregistrements des DAC. Le poids moyen d'une dose a été mesuré 2 fois par semaine sur chaque DAC par pesée de 10 doses consécutives. Ces doses bi-hebdomadaires ont été conservées afin de constituer un échantillon moyen annuel représentatif $d u$ concentré distribué.

En période de pénurie fourragère, les quantités de fourrages complémentaires distribuées et refusées ont été pesées tous les jours afin de calculer les quantités moyennes ingérées par traitement.

\subsubsection{Analyses chimiques des aliments et féces}

Après pesée en vert, l'échantillon moyen d'herbe de chaque parcelle a été séché en étuve ventilée à $80^{\circ} \mathrm{C}$ pendant $24 \mathrm{~h}$ pour la détermination de la teneur en matière sèche (MS) puis broyée (grille de $0,5 \mathrm{~mm}$ ) afin de procéder au dosage des teneurs en MO, matières azotées totales (MAT : $\mathrm{N} \times 6,25$ ) et cellulose brute (CB). La digestibilité de la MO de l'herbe offerte (dHO) a été calculée à partir de la détermination de la digestibilité pepsine-cellulase et de l'équation publiée par Aufrère et Demarquilly [2].

La teneur en MO, MAT, CB, Extrait Ethéré (EE) et en constituants pariétaux (NDF-ADFADL) du concentré a été déterminée sur chaque échantillon moyen annuel broyé. Après passage à l'étuve et broyage, les teneurs en MO, N et $\mathrm{ADF}$ des échantillons de féces journaliers ont été déterminées.

L'ensemble de ces analyses chimiques a été réalisé au laboratoire de développement et d'analyses 22 (LDA - 22440 Ploufragan).

La valeur nutritive (UFL et PDI) des différents aliments a ensuite été calculée à partir des équations de prédiction proposées par l'Inra en 1987 [29].

\subsection{Calculs et analyses statistiques}

Compte tenu des conditions climatiques des 2 premières années, la synthèse des résultats, tant agronomiques que zootechniques, a été réalisée sur deux périodes :

- le printemps, composé uniquement du pâturage des prairies permanentes ( 3 cycles), disponible lors des 5 années d'expérience. Certaines années, entre le deuxième et troisième cycle, les animaux ont pâturé 1 ou 2 parcelles de prairies temporaires pendant 4 à $12 \mathrm{j}$. Afin de rester homogène entre années, ces brèves périodes ont été éliminées lors de l'analyse des performances individuelles de printemps.

- la saison complète (d'avril à octobre) qui intègre les résultats observés durant 5 cycles de pâturage lors des 3 dernières années d'expérience. Néanmoins, en septembre 1993, les vaches sont restées en stabulation durant $25 \mathrm{j}$ et ont été alimentées à l'identique pour les trois traitements. Cette courte période a été exclue des résultats. Pour ces 3 années, les bilans de pâturage ont alors été calculés selon la méthodologie proposée par Hoden et al. [25].

L'ensemble des analyses statistiques décrites ci-après a été réalisé à l'aide de la procédure GLM sous Sas [46], sauf pour les variations de note d'état analysées selon la procédure NPar l Way [46]. 


\subsubsection{Production des prairies et composition chimique de l'herbe offerte}

Pour l'ensemble des prélévements d'herbe, la biomasse en entrée de parcelle (BE en $\mathrm{kg}$ MS/ha) a été recalculée à une hauteur de fauche moyenne et constante de $4,5 \mathrm{~cm}$, en intégrant la densité DE et la hauteur HE de l'herbe mesurée à l'entrée sur l'ensemble de la parcelle, selon la formule : $\mathrm{BE}=\mathrm{DE} \times(\mathrm{HE}-4,5)$. $\mathrm{La}$ biomasse produite (BP en $\mathrm{kg} \mathrm{MS} / \mathrm{ha}$ ) qui intègre la biomasse accumulée pendant la repousse et celle durant la présence des animaux sur la parcelle a été calculée selon la méthode proposée en encadré 1 .

Les résultats de production fourragère, de composition chimique de l'herbe offerte et de sa valeur nutritive ont été analysés, en séparant les prairies permanentes des prairies temporaires, selon le modèle partiellement hiérar- chisé : $Y_{i j k}=A_{i}+P_{j}\left(A_{j}\right)+F_{k}+A_{i} \times F_{k}+e_{i j k}$ avec $\mathrm{A}=$ effet Année $(\mathrm{i}=1$ à 5 ou $\mathrm{i}=3$ à 5 ), $P(A)=$ effet entrée de Parcelle intra Année $(\mathrm{j}=1$ à 9 ou 15), $\mathrm{F}=$ effet Fertilisation $(\mathrm{k}=1$ à 3 ) et $\mathrm{e}=$ résiduelle.

\subsubsection{Performances animales et utilisation du couvert végétal}

Les performances laitières moyennes individuelles journalières et les quantités moyennes de concentré ingérées ont été calculées à partir des données enregistrées pour chaque vache durant le printemps ( 5 ans) ou toute la saison de pâturage ( 3 ans). Les taux butyreux et protéique moyens individuels ont été pondérés en calculant le rapport entre la quantité de matières et la quantité de lait produite lors des jours de mesures des taux.

Le poids vif moyen individuel a été calculé en utilisant l'ensemble des pesées réalisées chaque année. Les variations de poids obser-

Encadré 1. Calcul de la biomasse totale produite sur prairies pâturées et estimation des quantités d'herbe ingérées à partir des hauteurs et de la densité de l'herbe offerte.

Au pâturage, contrairement à la récolte par fauche, la biomasse produite au cours d'une repousse $\left(\mathrm{BP}_{\mathrm{i}}\right.$ en $\mathrm{kg}$ de $\left.\mathrm{MS} / \mathrm{ha}\right)$ ne correspond pas à la biomasse offerte à l'entrée de la parcelle, du fait d'une hauteur de sortie des animaux souvent différente de la hauteur de coupe de la motofaucheuse lors du prélévement, de l'accumulation de zones de refus au cours des cycles et de la croissance de l'herbe pendant le temps de séjour (TS ${ }_{i}$ en jour) des animaux [32].

Selon les principes décrits par Kanneganti et Kaffka [31], la biomasse nette accumulée durant le temps de repousses $\left(\mathrm{TR}_{\mathrm{i}}\right.$ en jour) est calculée par la formule $\mathrm{DE}_{\mathrm{i}} \times\left(\mathrm{HE}_{\mathrm{i}}-\mathrm{HS}_{\mathrm{i}-1}\right)$, en utilisant la hauteur d'herbe mesurée en sortie de parcelle ou après la fauche des refus (HS en $\mathrm{cm}$ ) au cycle [i-1]. Pour le premier cycle, la biomasse récoltée au dessus de $4,5 \mathrm{~cm}$ est définie par convention comme celle accumulée depuis le 15/03.

D'autre part, en pâturage tournant, la croissance du végétal en présence des animaux est calculée en admettant qu'elle représente $50 \%$ de celle observée durant le temps de repousses précédent. Cette valeur de $50 \%$, retenue par Hoden et al. [26], est proche de la valeur moyenne obtenue à partir de la formule modifiée de Linehan [33] si l'on admet une croissance linéaire de l'herbe pendant le temps de séjour des animaux. Après simplification, la formule proposée pour estimer la biomasse produite au cours d'un cycle [i] devient :

$$
\mathrm{BP}_{\mathrm{i}}=\mathrm{DE}_{\mathrm{i}} \times\left(\mathrm{HE}_{\mathrm{i}}-\mathrm{HS}_{\mathrm{i}-1}\right) \times\left(1+0,5 \times\left(\mathrm{TS}_{\mathrm{i}} / \mathrm{TR}_{\mathrm{i}}\right)\right) .
$$

La biomasse totale (BT en $\mathrm{kg} \mathrm{MS/ha)} \mathrm{produite} \mathrm{par} \mathrm{la} \mathrm{prairie} \mathrm{au} \mathrm{cours} \mathrm{de} \mathrm{l'année} \mathrm{corres-}$ pond alors à la somme des BP obtenues à chaque cycle.

Les quantités d'herbe ingérées (QHI, en $\mathrm{kg} \mathrm{MS} / \mathrm{vl} / \mathrm{j})$ sont ensuite calculées à partir des hauteurs mesurées à l'entrée et à la sortie de la parcelle comme l'ont proposé Hoden et al. [28], en y ajoutant toutefois la biomasse produite pendant le séjour des animaux sur la parcelle et considérée comme consommée par les vaches. Ainsi,

$$
\mathrm{QHI}=\mathrm{DE}_{\mathrm{i}} \times \mathrm{S}_{\mathrm{i}} \times\left[\left(\mathrm{HE}_{\mathrm{i}}-\mathrm{HS}_{\mathrm{i}}\right)+0,5 \times\left(\mathrm{HE}_{\mathrm{i}}-\mathrm{HS}_{\mathrm{i}-1}\right) / \mathrm{TR}_{\mathrm{i}}\right]
$$


vées lors des 3 dernières années ont été calculées par différence entre les 2 premières pesées consécutives suivant le début de l'expérience et les 2 dernières pesées consécutives réalisées en automne. Les variations de note d'état ont été estimées par différence entre la note initiale de mise en lot et la note finale attribuée à chaque vache en juillet ou en octobre.

L'effet du niveau de fertilisation azotée des prairies sur les performances zootechniques individuelles a été testé par analyse de covariance selon le modèle : $Y_{i i}=A_{i}+F_{j}+A_{i} \times F_{j}$ $+b \times \operatorname{Cov}_{i j}+e_{i j}$ avec $\mathrm{A}=$ effet Année $(i=1$ à 5 ou i $=3$ à 5$), \mathrm{F}=$ effet Fertilisation $(\mathrm{k}=1$ à 3$)$, $\mathrm{Cov}=$ valeur moyenne de la variable $\mathrm{Y}$ observée avant l'expérience et $\mathrm{e}=$ résiduelle. En l'absence d'interactions de pente, celles ci ont été éliminées du modèle d'analyse. Une analyse non paramétrique (Test des rangs de KruskalWallis) a permis de tester l'effet des traitements sur les variations de note d'état des animaux.

$\grave{A}$ partir des teneurs $\mathrm{N}$ et ADF ( $\% \mathrm{MO})$ des fèces, la digestibilité du régime ingéré par les 3 lots d'animaux a été estimée selon l'équation [39] :

$$
\begin{gathered}
\mathrm{dMOr}=0,778+0,0334 \times \mathrm{N}-0,0038 \\
\times \mathrm{ADF}+\mathrm{d} \\
\left(n=24-\mathrm{Syx}=0,013-\mathrm{R}^{2}=0,89\right)
\end{gathered}
$$

avec $d=+0,007$ au premier cycle, $+0,008$ sur les repousses de printemps et $-0,015$ en automne.

La digestibilité de la MO de l'herbe ingérée (dHI) a été calculée par la formule : $\mathrm{dHI}=$ (dMOr $-0,15 \times 0,868) / 0,85$ en admettant l'additivité des digestibilités des composants du régime et, qu'en moyenne, le concentré représentait $15 \%$ de l'ingéré total.

La digestibilité de l'herbe ingérée et la teneur en urée du lait mesurée en début ou en fin de parcelle a été analysée selon le modèle : $Y_{i j k}=A_{i}+C_{j}+F_{k}+A_{i} \times F_{k}+C_{j} \times F_{k}+e_{i j k}$ avec avec $\mathrm{A}=$ effet Année ( $\mathrm{i}=1 \mathrm{a} 4), \mathrm{C}=$ effet Cycle $(\mathrm{j}=1$ à 5$), \mathrm{F}=$ effet Fertilisation $(\mathrm{k}=1$ à 3 ) et $\mathrm{e}=$ résiduelle.

Afin d'apprécier les conditions de pâturage et l'utilisation de l'herbe par les animaux, différents paramètres caractérisant l'herbe offerte et ingérée ont été calculés pour chaque entrée de parcelle. Le volume d'herbe offert (VHO en $\mathrm{m}^{3} /$ vache/j) intègre la hauteur d'herbe (HE) au dessus de $4,5 \mathrm{~cm}$ et la surface offerte par vache et par jour ( $\mathrm{S}$ en $\left.\mathrm{m}^{2} / \mathrm{vl} / \mathrm{j}\right)$. La quantité d'herbe offerte (QHO en $\mathrm{kg} \mathrm{MS} / \mathrm{vl} / \mathrm{j})$ au dessus de $4,5 \mathrm{~cm}$ correspond alors au volume d'herbe offert multiplié par la densité de l'herbe (DE), auquel il convient d'ajouter la biomasse produite pendant le temps de séjour des animaux sur la parcelle (encadré l).

Les quantités d'herbe ingérées (QHI en $\mathrm{kg}$ $\mathrm{MS} / \mathrm{vl} / \mathrm{j}$ ) ont été estimée, selon la méthode de calcul détaillée à l'encadré 1 . Les quantités totales ingérées (QTI en $\mathrm{kg} \mathrm{MS} / \mathrm{vl} / \mathrm{j}$ ) correspondent finalement à la somme de l'herbe, du concentré et des éventuels fourrages complémentaires ( 0,8 à $1,0 \mathrm{~kg}$ MS en moyenne) ingérés par vache et par jour sur une parcelle.

L'ensemble de ces paramètres caractérisant l'utilisation de la prairie par les animaux au printemps sur prairies permanentes ( 5 ans) ou durant la saison complète de pâturage ( 3 ans) a été analysé à partir d'un modèle hiérarchisé identique à celui développé pour l'analyse de la productivité des prairies.

\section{RÉSULTATS}

\subsection{Conditions climatiques et déroulement du pâturage}

Les 2 premières années d'expérience se sont caractérisées par un déficit hydrique important durant les 3 mois d'été ( -92 et $-68 \mathrm{~mm}$ respectivement en 1990 et 91 par rapport à la moyenne des 25 années - tableau I), associé en 1990 à de très faibles pluies en mai. De plus, les températures moyennes de juillet et août ont été nettement supérieures à la moyenne 1965-89. À l'inverse, les années 1992 et 1994 ont présenté des séquences climatiques très favorables à la croissance de l'herbe : un printemps relativement chaud $\left(+1,5\right.$ et $+0,8^{\circ} \mathrm{C}$ en mai et juin), une pluviométrie régulière et abondante en été. L'année 1993 a été en moyenne trés pluvieuse $(+118 \mathrm{~mm}$ en 7 mois par rapport aux 25 années) mais a présenté un déficit de pluviométrie très marqué en août $(-33 \mathrm{~mm})$ qui a interrompu l'expérience durant 3 semaines en septembre.

En moyenne, sur 5 années, la mise à l'herbe a eu lieu le 03/04 ( $\pm 5 \mathrm{j}$ ). Les deux premiers cycles ont eu une durée moyenne 
cumulée de $72 \mathrm{j}$, y compris la période de transition (tableau II). Lors du troisième cycle, les prairies temporaires, récoltées en ensilage le 25/05, ont été introduites simultanément dans les 3 traitements. Les troisième et quatrième cycles d'une durée moyenne équivalente (44 à 48 j) ont utilisé le maximum de surfaces pâturées. Le troi- sième cycle a été caractérisé par une durée très variable $( \pm 8 j)$, ce qui traduit la variabilité inter annuelle importante de la croissance de l'herbe à cette période. Le dernier pâturage, écourté à la fois par les premiers tarissements et les conditions climatiques, valorise surtout les prairies permanentes. Lors des 3 dernières années, la saison com-

Tableau I. Température et pluviométrie mensuelles observées au cours des 5 années d'expérience.

\begin{tabular}{lcccccccc}
\hline & & Avril & Mai & Juin & Juilt & Aôut & Sept & Oct \\
\hline Température $\left({ }^{\circ} \mathrm{C}\right)$ & 1990 & 7,5 & 12,7 & 13,0 & 17,5 & 18,2 & 12,8 & 11,9 \\
& 1991 & 7,2 & 9,7 & 12,1 & 16,5 & 17,4 & 15,1 & 9,5 \\
& 1992 & 7,5 & 12,9 & 15,1 & 16,9 & 17,0 & 13,4 & 8,2 \\
& 1993 & 9,8 & 12,9 & 15,8 & 15,3 & 14,7 & 12,6 & 8,8 \\
\multirow{2}{*}{ Moyenne } & 1994 & 8,1 & 12,2 & 15,3 & 19,4 & 16,9 & 13,4 & 10,3 \\
Pluviométrie (mm) & $1965-89$ & 7,6 & 10,9 & 14,4 & 16,3 & 15,9 & 13,9 & 10,4 \\
& 1991 & 72,8 & 10,6 & 56,9 & 12,3 & 15,2 & 37,3 & 64,4 \\
& 1992 & 46,9 & 41,2 & 86,2 & 32,4 & 17,9 & 38,1 & 67,2 \\
& 1993 & 100,9 & 75,4 & 83,2 & 36,2 & 10,3 & 134,8 & 67,5 \\
& 1994 & 89,8 & 69,6 & 44,9 & 88,0 & 77,3 & 99,5 & 48,1 \\
Moyenne & $1965-89$ & 49,0 & 71,2 & 49,5 & 52,2 & 43,4 & 61,2 & 63,8 \\
\hline
\end{tabular}

Comparaison avec la moyenne de 25 années (1965-1989).

Tableau II. Durée de pâturage et surface totale pâturée à chaque cycle selon les traitements.

\begin{tabular}{lccccc}
\hline $\begin{array}{l}N^{\circ} \text { cycle } \\
\text { Date début }\end{array}$ & 1 & 2 & 3 & 4 & 5 \\
\hline $\begin{array}{l}\text { Durée totale (j) } \\
\text { dont prairies temporaires }\end{array}$ & $03 / 04$ & $11 / 05$ & $15 / 06$ & $10 / 08$ & $24 / 09$ \\
& $37 \pm 5$ & $35 \pm 4$ & $48 \pm 8$ & $44 \pm 1$ & $24 \pm 4$ \\
$\begin{array}{l}\text { Surface totale pâturée (ares/vache) } \\
\text { dont Prairies temporaires }\end{array}$ & 0 & 3 & 20 & 21 & 5 \\
$\quad$ Haut & $19 \pm 1,2$ & $24 \pm 3,6$ & $36 \pm 5,1$ & $40 \pm 0,0$ & $27 \pm 6,7$ \\
Moyen & 0 & 4 & 16 & 20 & 7 \\
Bas & $24 \pm 2,8$ & $30 \pm 4,0$ & $45 \pm 6,0$ & $48 \pm 1,7$ & $34 \pm 8,0$ \\
& 0 & 4 & 19 & 22 & 8 \\
& $30 \pm 3,4$ & $36 \pm 4,9$ & $52 \pm 8,2$ & $57 \pm 2,6$ & $42 \pm 9,3$ \\
\hline
\end{tabular}

Les résultats des trois premiers cycles ont été calculés sur 5 années, ceux des deux derniers cycles sur les 3 dernières années d'expérience. Les écarts types correspondent à la variabilité inter annuelle. 
plète de pâturage a duré environ $190 \mathrm{j}$ dont 142 sur prairies permanentes. Le temps de séjour par parcelle a été en moyenne de $12 \mathrm{j}$ au printemps et de $6 \mathrm{j}$ environ en automne.

Au printemps, la surface de prairies permanentes affectée à chaque traitement, respectivement 20,26 et 32 ares/vache pour les traitements $\mathrm{H}, \mathrm{M}$ et B, n'a pas été totalement pâturée chaque année (tableau II). Deux années sur 5, l'excédent de surface a été ensilé en fin de premier cycle $(5 / 05)$. De même, la surface maximale pâturée moyenne (quatrième cycle) des traitements Moyen et Bas est restée un peu inférieure à celle affectée ( -2 et -3 ares/vache). Globalement, au cours de cette expérience, la réduction ou la suppression de la fertilisation azotée a entraîné un accroissement des surfaces pâturées à chaque cycle de respectivement 20 à $25 \%$ pour le traitement Moyen et 45 à $55 \%$ pour le traitement Bas, comparativement au traitement Haut.

\subsection{Production des prairies pâturées et composition de l'herbe offerte}

À même âge de repousses, la réduction de la fertilisation azotée sur prairies permanentes (M vs $\mathrm{H}$ - tableau III) a entraîné une réduction de la biomasse $(-310 \mathrm{~kg}$ $\mathrm{MS} / \mathrm{ha}, p<0,001$ ) et de la hauteur de l'herbe à l'entrée de parcelle $(-1,0 \mathrm{~cm}$, $p<0,001)$. La suppression des apports d'azote ( $\mathrm{B}$ vs $\mathrm{H}$ ) a accentué ces écarts qui atteignent alors $-780 \mathrm{~kg}$ et $-2,6 \mathrm{~cm}$ $(p<0,001)$. En moyenne par cycle, au cours des 5 années, la biomasse des prairies $\mathrm{B}$ et $\mathrm{M}$ a représenté 65 et $84 \%$ de celle du traitement $\mathrm{H}$.

Entre années, des différences d'amplitudes dans les effets de la fertilisation azotée sont apparues, mais n'ont pas modifié la hiérarchie des traitements (Interaction année $\times$ traitement, $p<0,01$ - figure l). La répétition des mêmes traitements sur les parcelles durant 5 années consécutives n'a entraîné aucun effet cumulatif défavorable sur la production et la composition floristique des prairies permanentes (D. Leconte, comm. pers.)

La réduction de la fertilisation azotée a entraîné un accroissement de la teneur en MS de l'herbe offerte (respectivement $+2,9$ et $+4,2$ points sur les traitements $M$ et $\mathrm{B}$ comparé à $\mathrm{H}, p<0,001)$ et une diminution de la teneur en MAT $(-48$ et -67 $\mathrm{g} / \mathrm{kg} \mathrm{MS}, p<0,001)$ et de la dHO $(-0,03$ et $-0,04, p<0,001)$. La diminution de la teneur en MAT a été plus sensible entre les traitements $\mathrm{H}$ et $\mathrm{M}$ que $\mathrm{B}$ et $\mathrm{M}$. Bien que significatif, l'effet de la fertilisation azotée sur la teneur en MO et CB a été modeste (tableau III). Aucune interaction année $x$ traitement n'a été mise en évidence sur l'ensemble des paramètres de composition chimique analysés.

En été-automne, sur prairies temporaires, la biomasse et la hauteur en entrée de parcelle ont été en moyenne plus élevées sur les prairies du traitement $H$ par rapport à $\mathrm{M}$ et $\mathrm{B}$ (respectivement +540 $\mathrm{kg} \mathrm{MS} /$ ha et $+1,6 \mathrm{~cm}, p<0,001)$. La contribution pondérale du trèfle blanc a été très faible sur les prairies du traitement $\mathrm{H}$ où elle $n$ 'a représenté en moyenne que $5 \%$ de la biomasse, avec une absence totale en dernière année d'expérience. Cette contribution a atteint en moyenne 26 et $28 \%$ sur les prairies $M$ et B. Elle a été maximale au troisième cycle (30 à $35 \%$ ) pour décroître de 6 à 10 points par cycle ensuite.

Les différents niveaux de fertilisation appliqués ont eu des effets beaucoup moins prononcés sur la composition chimique de l'herbe des prairies temporaires (tableau III). En particulier, la teneur en MAT et la dHO de l'herbe ont été un peu plus élevées (respectivement $+8,5 \mathrm{~g} / \mathrm{kg}$ MS et $+0,015, p<0,01)$ sur les traitements $\mathbf{M}$ et $\mathbf{B}$ que $\mathrm{H}$. Sur les prairies $\mathrm{M}$ et $B$, ne recevant aucune fertilisation après la récolte en ensilage, chaque point de 


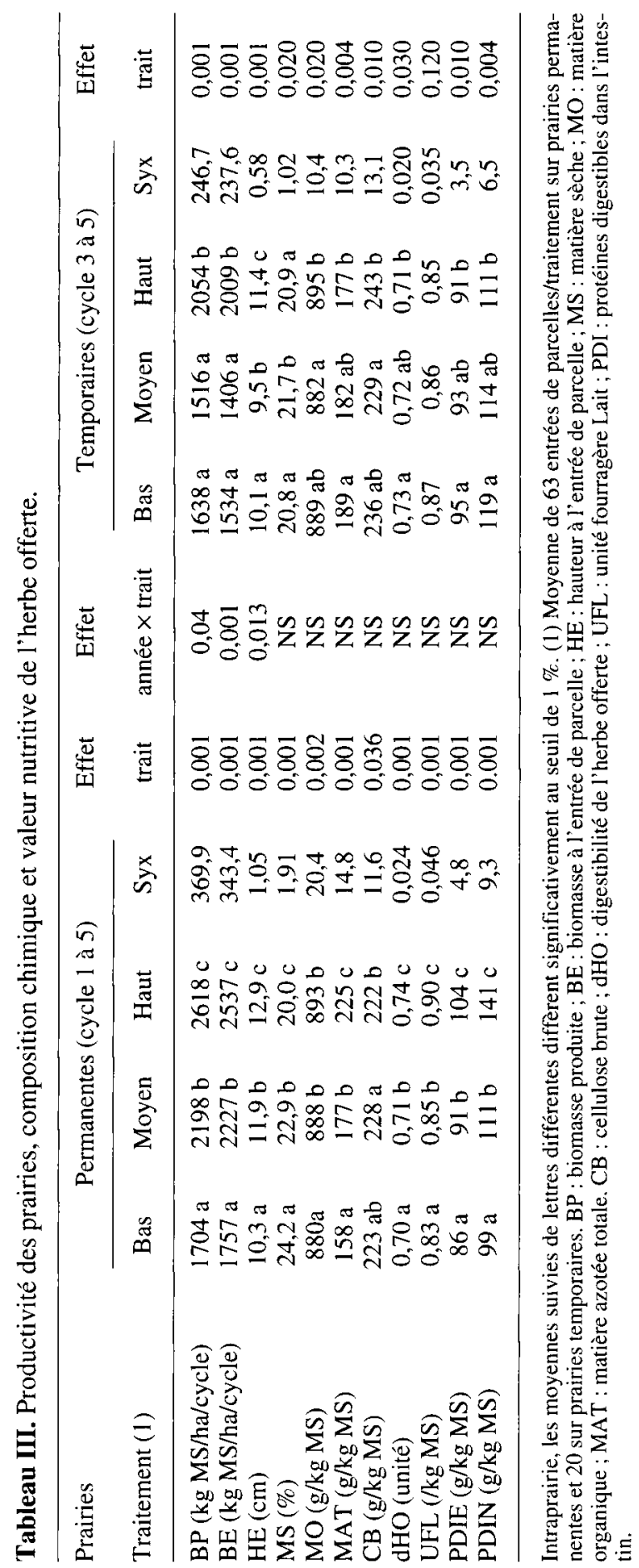


trèfle blanc en plus dans la biomasse récoltée a entraîné, en moyenne à chaque cycle, une augmentation de $0,85 \mathrm{~g}$ de MAT par $\mathrm{kg}$ de $\mathrm{MS}$ et de $0,8410^{-3}$ de digestibilité, une réduction de $0,68 \mathrm{~g}$ de $\mathrm{CB}$ par $\mathrm{kg}$ de MS et a accru la biomasse de $22,5 \mathrm{~kg}$ MS/ha $(p<0,001)$.

\subsection{Performances zootechniques et utilisation du couvert végétal}

Lors des calculs réalisés sur les cinq printemps ou les trois dernières années, 265 et 161 vaches ont été respectivement retenues. En effet, deux vaches, une en 1991 (traitement Bas) et l'autre en 1992 (traitement Haut) ont été éliminées en cours d'expérience pour raisons sanitaires (mammite, accident). D'autre part, trois vaches (deux en 1990 appartenant au traitement Moyen et une du traitement Bas en 1991) caractérisées par un stade de lactation très précoce en début d'expérience (< à 30 j) ont été éliminées lors du dépouillement compte tenu de leur période de référence instable et de l'évolution spé- cifique de leur courbe de lactation au cours du printemps.

\subsubsection{Printemps (5 années)}

En moyenne, la durée de la période expérimentale a été de $85 \mathrm{j}( \pm 5)$ de pâturage. Les animaux ont produit $27,4 \mathrm{~kg}$ de lait brut, avec un taux butyreux de 38,6 $\mathrm{g} / \mathrm{kg}$ et un taux protéique de $29,7 \mathrm{~g} / \mathrm{kg}$ et ont ingéré $2,20 \mathrm{~kg}$ de MS de concentré. La réduction simultanée de la fertilisation azotée et du chargement n'a pas eu d'effet significatif sur la production de lait brut et $4 \%$, le taux butyreux et les quantités de matières grasses et protéiques (tableau $I V$ ). Cependant le taux protéique moyen a été plus élevé chez les animaux des traitements Moyen et Bas (+ 0,5 , $p<0,003)$. Bien que les écarts entre traitements soient faibles, les animaux du traitement B ont eu un poids vif moyen supérieur $(p<0,005)$ à ceux des traitements $M$ et $H$. Les pertes d'état $(-0,15$ pt en moyenne) n'ont pas été différentes entre traitements.

Tableau IV. Performances zootechniques individuelles journalières réalisées durant le printemps sur prairies permanentes ( 5 années).

\begin{tabular}{lcccccc} 
Traitement & $\begin{array}{c}\text { Bas } \\
(n=88)\end{array}$ & $\begin{array}{c}\text { Moyen } \\
(n=88)\end{array}$ & $\begin{array}{c}\text { Haut } \\
(n=89)\end{array}$ & Syx & $\begin{array}{c}\text { Effet } \\
\text { trait }\end{array}$ & $\begin{array}{c}\text { Effet } \\
\text { année } \times \text { trait }\end{array}$ \\
\hline Concentré ingéré $(\mathrm{kg} \mathrm{MS})$ & 2,1 & 2,1 & 2,3 & & & \\
Lait $(\mathrm{kg})$ & 27,3 & 27,3 & 27,5 & 1,63 & $\mathrm{NS}$ & $\mathrm{NS}$ \\
Lait $4 \%(\mathrm{~kg})$ & 26,7 & 26,7 & 26,7 & 1,66 & $\mathrm{NS}$ & 0,120 \\
Taux butyreux $(\mathrm{g} / \mathrm{kg})$ & 38,9 & 38,6 & 38,3 & 2,38 & $\mathrm{NS}$ & $\mathrm{NS}$ \\
Taux protéique $(\mathrm{g} / \mathrm{kg})$ & $29,9 \mathrm{a}$ & $29,8 \mathrm{a}$ & $29,4 \mathrm{~b}$ & 0,95 & 0,003 & $\mathrm{NS}$ \\
Matières grasses $(\mathrm{g})$ & 1056 & 1049 & 1048 & 77,3 & $\mathrm{NS}$ & $\mathrm{NS}$ \\
Matières protéiques $(\mathrm{g})$ & 807 & 806 & 802 & 49,0 & $\mathrm{NS}$ & 0,012 \\
Poids moyen $(\mathrm{kg})$ & $635 \mathrm{a}$ & $632 \mathrm{ab}$ & $627 \mathrm{~b}$ & 15,4 & 0,005 & $\mathrm{NS}$ \\
Var. note d'état $(\mathrm{pt})$ & $-0,26$ & $-0,09$ & $-0,06$ & & $\mathrm{NS}$ & \\
& & & & & & \\
Urée du lait $(\mathrm{mg} / 100 \mathrm{~mL})(1)$ & $32,9 \mathrm{a}$ & $43,2 \mathrm{~b}$ & $52,3 \mathrm{c}$ & 7,11 & 0,001 & $\mathrm{NS}$ \\
$\quad$ Début de parcelle & $25,7 \mathrm{a}$ & $32,8 \mathrm{~b}$ & $40,0 \mathrm{c}$ & 3,97 & 0,001 & $\mathrm{NS}$ \\
$\quad$ Fin de parcelle & & & & & & $\mathrm{N}$
\end{tabular}

Les moyennes suivies de lettres différentes diffèrent significativement au seuil de $1 \%$. (1) Moyenne de onze débuts et neuf fins de parcelle/traitement. 
La hiérarchie entre traitements a été différente selon les années pour la production de matières protéiques $(p<0,012)$ et dans une moindre mesure pour la production de lait $4 \%$ (figure 2). Lors de 2 années, en 1990 par rapport au traitement Moyen et en 1994 par rapport au traitement Haut, les animaux du traitement Bas ont produit moins de protéines par jour (-34 g). À l'inverse, en 1992, ces animaux ont produit $43 \mathrm{~g}$ de protéines en plus que les animaux des deux autres traitements.

La teneur en urée des laits de mélange, en début comme en fin de parcelle, a été significativement diminuée par la réduction de fertilisation azotée $(p<0,001)$. En fin de parcelle, la teneur en urée du lait a été systématiquement plus faible et les écarts entre traitements ont été moins importants qu'en début de parcelle.

Au printemps, la hauteur de l'herbe offerte sur les traitements $M$ et $B$, respectivement 13,0 et $11,1 \mathrm{~cm}$, a été inférieure à celle du traitement $\mathrm{H}(14,0 \mathrm{~cm}-$ $p<0,001)$. En revanche, la surface offerte journalière a été accrue de 16 et $34 \mathrm{~m}^{2} / \mathrm{vl} / \mathrm{j}$ sur les traitements Moyen et Bas (tableau V). Dans ces conditions, les quantités d'herbe offerte et la hauteur après pâturage ont été un peu plus élevées sur le traitement Moyen $(p<0,001)$ et n'ont pas différé significativement entre les traitements Haut et Bas.

Les quantités d'herbe ingérées calculées ont été inférieures sur le traitement Bas $(-1,4 \mathrm{~kg}$ MS, $p<0,005)$ et n'ont pas différé entre les traitements Moyen et Haut. La digestibilité de l'herbe ingérée par les animaux a été de 0,78 en moyenne et n'a pas significativement varié avec le niveau de fertilisation azotée. Elle a été systématiquement plus élevée d'environ 0,05 unité que celle mesurée sur l'herbe offerte des mêmes parcelles (tableau $V$ ).

Les moyennes sur 5 années masquent néanmoins des réponses aux traitements variables selon les années (Interaction année $\times$ traitement, $p<0,01-$ figure 1 ). En particulier, en 1990 et 1994, le traitement Bas se caractérise par des hauteurs moyennes à l'entrée très faibles et inférieures à $10 \mathrm{~cm}$ tandis que les écarts de surface offerte entre traitements sont maximum. Les hauteurs en sortie de parcelle ont également été les plus faibles sur le traitement Bas en $1990(5,7 \mathrm{~cm})$ et 1994 $(5,8 \mathrm{~cm})$ alors qu'elles sont plus élevées en 1992 pour les traitements Moyen et Bas (respectivement 7,0 et $6,8 \mathrm{~cm}$ ). En revanche, sur les critères de valorisation de l'herbe, qui intègrent dans leur calcul la présence de l'animal (QTI ou dHI), aucune interaction ne s'est révélée significative $(p>0,10)$.

\subsubsection{Saison complète (3 années)}

Sur l'ensemble des paramètres zootechniques étudiés, aucune différence significative liée à l'application des traitements n'a été mise en évidence (tableau VI). Les animaux ont produit $22,1 \mathrm{~kg}$ de lait contenant $38,9 \mathrm{~g} / \mathrm{kg}$ de taux butyreux et $31,2 \mathrm{~g} / \mathrm{kg}$ de taux protéique. Les quantités journalières moyennes de concentré ingérées ont été de 2,05 kg MS. Toutefois, les vaches du traitement Bas ont eu tendance à produire un peu plus de matières grasses et protéiques $(p<0,11)$ et un lait plus riche en protéines $(p<0,07)$ que les vaches des autres traitements. Les animaux ont repris en moyenne $265 \mathrm{~g} / \mathrm{j}$ mais ont perdu $0,15 \mathrm{pt}$ de note d'état pour aboutir à un poids final de $666 \mathrm{~kg}$. Aucune interaction significative entre les années et les traitements n'a été mise en évidence.

La teneur en urée du lait produit sur prairies permanentes au cours des cinq cycles a été plus élevée en début qu'en fin de parcelle et s'est accrue linéairement avec le niveau de fertilisation azotée (tableau VI).

Le volume et les quantités d'herbe offertes ont été similaires entre les trois traitements (respectivement $6,7 \mathrm{~m}^{3}$ et 22,1 

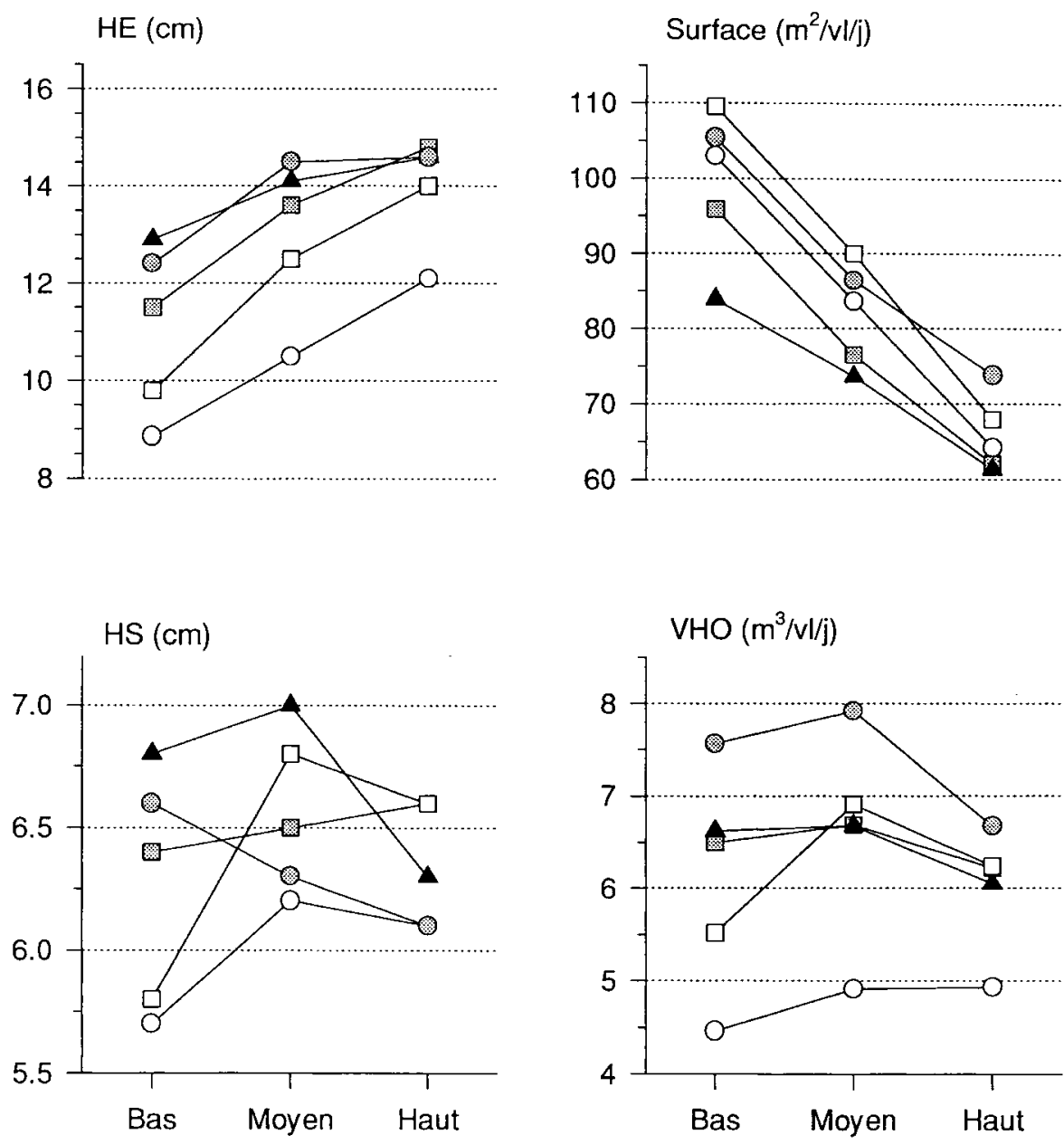

Fertilisation - Chargement

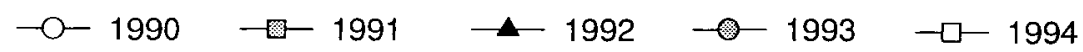

Figure 1. Variations interannuelles de l'effet de la fertilisation azotée et du chargement sur l'utilisation des prairies permanentes.

$\mathrm{kg} \mathrm{MS,} p>0,50$ ). Malgré une hauteur en sortie de parcelle un peu plus élevée sur le traitement $\mathrm{H}(p<0,003)$, les quantités d'herbe ingérées n'ont pas différé significativement entre traitements (tableau VII). En moyenne, sur les 3 années, les animaux ont ingéré 21 à 22 $\mathrm{kg}$ de MS dont 18,5 kg MS d'herbe. La digestibilité de l'herbe ingérée n'a pas été affectée par les traitements contrairement 

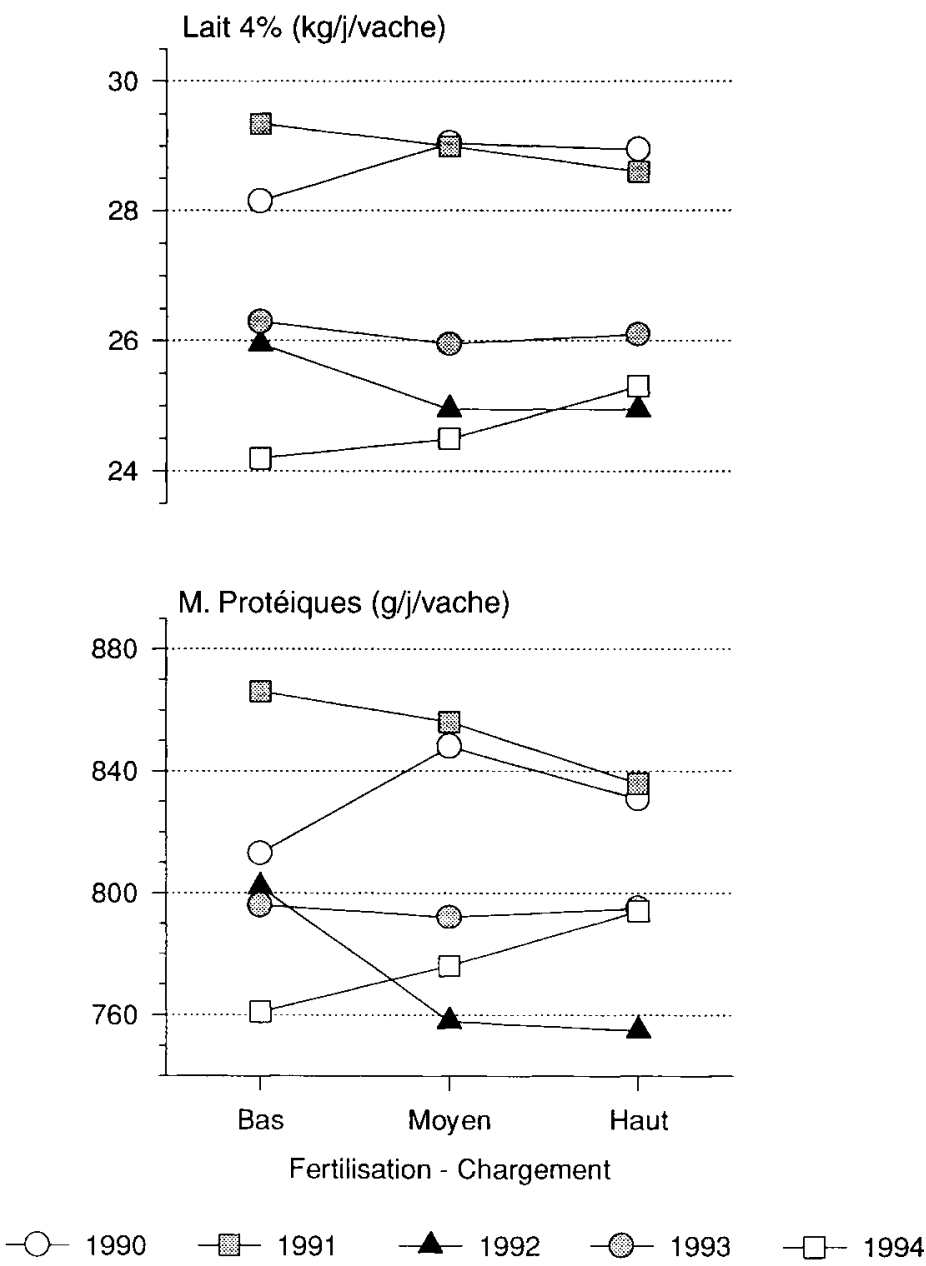

Figure 2. Variations interannuelles de l'effet de la fertilisation azotée et du chargement sur la production de lait $4 \%$ et de protéines (moyennes ajustées intra-année)

à celle de l'herbe offerte $(p<0,021)$. Elle a été en moyenne de 0,76 sur l'ensemble des prairies permanentes et temporaires.

L'effet de la fertilisation azotée a été variable selon les années sur les paramètres caractérisant l'herbe offerte aux animaux $(p<0,01)$. Cette interaction traduit en fait une inversion des effets entre les traitements $\mathrm{H}$ et B lors des années 1992 et 1994 , avec des valeurs de volume et quantités d'herbe offertes plus élevées pour le lot Bas en 1992. Les écarts interannuels ont été toutefois plus modérés qu'au printemps, sans doute du fait de l'introduction des prairies temporaires à partir du troisième cycle. Aucune interaction significative n'a été mise en évidence sur les quantités d'herbe $(p<0,07)$ ou totales $(p<0,14)$ ingérées. 
Tableau V. Valorisation du couvert végétal au cours du printemps sur prairies permanentes ( 5 ans).

\begin{tabular}{|c|c|c|c|c|c|c|}
\hline Traitement (1) & Bas & Moyen & Haut & Syx & $\begin{array}{l}\text { Effet } \\
\text { trait }\end{array}$ & $\begin{array}{c}\text { Effet } \\
\text { année } \times \text { trai }\end{array}$ \\
\hline BE (kg MS/ha) & $1947 \mathrm{a}$ & $2492 \mathrm{~b}$ & $2800 \mathrm{c}$ & 329 & 0,001 & 0,005 \\
\hline $\mathrm{HE}(\mathrm{cm})$ & $11,1 \mathrm{a}$ & $13,0 \mathrm{~b}$ & $14,0 \mathrm{c}$ & 1,05 & 0,001 & 0,051 \\
\hline Surface offerte $\left(\mathrm{m}^{2} / \mathrm{vl} / \mathrm{j}\right)$ & $99,6 \mathrm{a}$ & $82,0 \mathrm{~b}$ & $65,8 \mathrm{c}$ & 6,8 & 0,001 & 0,015 \\
\hline $\mathrm{VHO}\left(\mathrm{m}^{3} / \mathrm{vl} / \mathrm{j}\right)$ & 6,1 a & $6,6 \mathrm{~b}$ & $6,0 \mathrm{a}$ & 0,68 & 0,001 & 0,013 \\
\hline QHO (kg MS/vl/j) (2) & $20,9 \mathrm{a}$ & $22,4 \mathrm{~b}$ & 20,5 a & 2,79 & 0,010 & 0,012 \\
\hline HS (cm) & $6,3 \mathrm{a}$ & $6,6 \mathrm{~b}$ & $6,4 \mathrm{a}$ & 0,40 & 0,009 & 0,001 \\
\hline QHI (kg MS/vl/j) & $15,9 \mathrm{a}$ & $17,6 \mathrm{~b}$ & $17,0 \mathrm{~b}$ & 2,11 & 0,004 & 0,110 \\
\hline QTI (kg MS/vl/j) & $19,2 \mathrm{a}$ & $20,6 \mathrm{~b}$ & $20,3 \mathrm{~b}$ & 1,98 & 0,007 & 0,090 \\
\hline dHO (unité) & $0,72 \mathrm{a}$ & $0,73 \mathrm{a}$ & $0,75 \mathrm{~b}$ & 0,029 & 0,011 & NS \\
\hline dHI (unité) & $0,77 \mathrm{a}$ & $0,78 a b$ & $0,79 \mathrm{~b}$ & 0,019 & 0,073 & NS \\
\hline
\end{tabular}

Les moyennes suivies de lettres différentes diffèrent significativement au seuil de $1 \%$. (1) Moyenne de 40 entrées de parcelles/traitement sur prairies permanentes, sauf pour la digestibilité de l'herbe offerte et ingérée qui repose sur 14 mesures/traitement. (2) Y compris la biomasse produite pendant le séjour des animaux sur la parcelle. BE : biomasse à l'entrée de parcelle; HE : hauteur à l'entrée de parcelle ; VHO : volume d'herbe offert; QHO : quantité d'herbe offerte ; HS : hauteur en sortie de parcelle ; QHI : quantité d'herbe ingérée ; QTI : quantité totale ingérée ; $\mathrm{dHO}$ : digestibilité de l'herbe offerte ; dHI : digestibilité de l'herbe ingérée.

Tableau VI. Performances zootechniques individuelles journalières réalisées durant la saison compléte de pâturage ( 3 années).

\begin{tabular}{lcccccc}
\hline Traitement & $\begin{array}{c}\text { Bas } \\
(n=54)\end{array}$ & $\begin{array}{c}\text { Moyen } \\
(n=54)\end{array}$ & $\begin{array}{c}\text { Haut } \\
(n=53)\end{array}$ & $\begin{array}{c}\text { Syx } \\
\text { trait }\end{array}$ & $\begin{array}{c}\text { Effet } \\
\text { année } \times \text { trait }\end{array}$ \\
\hline Concentré ingéré $(\mathrm{kg} \mathrm{MS})$ & 2,0 & 2,0 & 2,1 & & & \\
Lait $(\mathrm{kg})$ & 22,4 & 21,8 & 22,0 & 2,09 & $\mathrm{NS}$ & $\mathrm{NS}$ \\
Lait 4\% $(\mathrm{kg})$ & 22,0 & 21,6 & 21,4 & 2,07 & $\mathrm{NS}$ & $\mathrm{NS}$ \\
Taux butyreux $(\mathrm{g} / \mathrm{kg})$ & 39,3 & 38,9 & 38,6 & 2,32 & $\mathrm{NS}$ & $\mathrm{NS}$ \\
Taux protéique $(\mathrm{g} / \mathrm{kg})$ & $31,5 \mathrm{a}$ & $31,2 \mathrm{ab}$ & $31,0 \mathrm{~b}$ & 1,19 & 0,080 & $\mathrm{NS}$ \\
Matières grasses $(\mathrm{g})$ & 871 & 839 & 840 & 87,8 & 0,120 & $\mathrm{NS}$ \\
Matières protéiques $(\mathrm{g})$ & 697 & 674 & 676 & 63,6 & 0,110 & $\mathrm{NS}$ \\
Poids moyen $(\mathrm{kg})$ & 645 & 644 & 639 & 18,4 & $\mathrm{NS}$ & $\mathrm{NS}$ \\
Poids final $(\mathrm{kg})$ & 666 & 669 & 662 & 32,5 & $\mathrm{NS}$ & $\mathrm{NS}$ \\
Var. poids vif(g/j) & 245 & 293 & 259 & 172 & $\mathrm{NS}$ & $\mathrm{NS}$ \\
Var. note d'état $(\mathrm{pt})$ & $-0,21$ & $-0,15$ & $-0,09$ & & $\mathrm{NS}$ & \\
& & & & & & \\
Urée du lait $(\mathrm{mg} / 100 \mathrm{~mL})(1)$ & & & & & & \\
$\quad$ Début de parcelle & $36,4 \mathrm{a}$ & $44,2 \mathrm{~b}$ & $51,9 \mathrm{c}$ & 4.57 & 0,001 & $\mathrm{NS}$ \\
$\quad$ Fin de parcelle & $28,6 \mathrm{a}$ & $35,0 \mathrm{~b}$ & $39,8 \mathrm{c}$ & 3,40 & 0,001 & $\mathrm{NS}$
\end{tabular}

Les moyennes suivies de lettres différentes diffèrent significativement au seuil de $1 \%$. (1) Moyenne de 14 débuts et 11 fins de parcelle de prairies permanentes/traitement. 
Tableau VII. Valorisation du couvert végétal sur l'ensemble de la saison de pâturage ( 3 ans).

\begin{tabular}{lcccccc}
\hline Traitement (1) & Bas & Moyen & Haut & $\begin{array}{c}\text { Effet } \\
\text { trait }\end{array}$ & $\begin{array}{c}\text { Effet } \\
\text { année } \times \text { trait }\end{array}$ \\
\hline BE (kg MS/ha) & $1750 \mathrm{a}$ & $2096 \mathrm{~b}$ & $2457 \mathrm{c}$ & 340 & 0,001 & 0,001 \\
HE (cm) & $10,5 \mathrm{a}$ & $11,5 \mathrm{~b}$ & $12,7 \mathrm{c}$ & 0,98 & 0,001 & 0,002 \\
Surface offerte $\left(\mathrm{m}^{2} / \mathrm{vl} / \mathrm{j}\right)$ & $125,1 \mathrm{a}$ & $105,3 \mathrm{~b}$ & $85,8 \mathrm{c}$ & 12,2 & 0,001 & $\mathrm{NS}$ \\
$\mathrm{VHO}\left(\mathrm{m}^{3} / \mathrm{vl} / \mathrm{j}\right)$ & 6,8 & 6,7 & 6,6 & 0,97 & $\mathrm{NS}$ & 0,002 \\
QHO (kg MS/vl/j) (2) & 21,9 & 22,2 & 21,7 & 3,90 & $\mathrm{NS}$ & 0,004 \\
& & & & & & \\
HS (cm) & $5,7 \mathrm{a}$ & $5,7 \mathrm{a}$ & $6,0 \mathrm{~b}$ & 0,51 & 0,003 & 0,001 \\
QHI (kg MS/vl/j) & 18,0 & 18,6 & 18,0 & 2,69 & $\mathrm{NS}$ & 0,070 \\
QTI (kg MS/vl/j) & 21,3 & 21,8 & $2 \mathrm{l}, 4$ & 2,57 & $\mathrm{NS}$ & 0,140 \\
dHO (unité) & $0,71 \mathrm{a}$ & $0,72 \mathrm{ab}$ & $0,74 \mathrm{~b}$ & 0,029 & 0,021 & $\mathrm{NS}$ \\
dHI (unité) & 0,76 & 0,77 & 0,77 & 0,022 & $\mathrm{NS}$ & $\mathrm{NS}$ \\
\hline
\end{tabular}

Les moyennes suivies de lettres différentes diffèrent significativement au seuil de $1 \%$. (1) Moyenne de 61 entrées de parcelles/traitement dont 20 sur prairies temporaires, sauf pour la digestibilité de l'herbe offerte et ingérée qui repose sur 16 mesures/traitement dont 5 sur prairies temporaires. (2) Y compris la biomasse produite pendant le séjour des animaux sur la parcelle. $\mathrm{BE}$ : biomasse à l'entrée de parcelle ; HE : hauteur à l'entrée de parcelle ; VHO : volume d"herbe offert; QHO : quantité d'herbe offerte ; HS : hauteur en sortie de parcelle ; QHI : quantité d’herbe ingérée ; QTI : quantité totale ingérée ; dHO : digestibilité de l’herbe offerte ; $\mathrm{dHI}$ : digestibilité de l'herbe ingérée.

\subsection{Bilan global de pâturage (3 années)}

Par rapport au traitement Haut, les traitements Moyen et Bas ont entraîné une réduction du nombre de journées de pâturage réalisé par hectare total du système (JP/ha), de respectivement 87 et $149 \mathrm{j}$ (tableau VIII). Les traitements Moyen et Bas ont produit respectivement 20 et $30 \%$ de lait en moins par hectare de prairies pâturées, pour des consommations de concentré et fourrages complémentaires par hectare inférieures de 22 et $33 \%$. La quantité de fourrage récoltée par hectare sur ces surfaces $(3,1$ t MS/ha) a été peu différente entre traitements du fait surtout d'une productivité élevée des prairies temporaires d'association RGA-TB des traitements Moyen et Bas.

Le lait $4 \%$ autonome par hectare total, correspondant au lait produit après déduction du lait équivalent à l'énergie apportée par les aliments complémentaires [25], a été inférieur de $1000 \mathrm{~kg}$ sur le traitement Moyen par rapport au Haut $(6500 \mathrm{~kg})$. Le traitement Bas a permis de produire $4800 \mathrm{~kg}$ de lait $4 \%$ autonome par hectare total, soit $1700 \mathrm{~kg}$ de moins que le traitement Haut.

\section{DISCUSSION}

Lors de cette expérience pluri-annuelle, la réduction simultanée de la fertilisation azotée et du chargement sans modification de la conduite alimentaire du troupeau a permis aux animaux de valoriser plus de surfaces au pâturage et de maintenir leurs performances moyennes individuelles. Ce résultat a été rendu possible grâce au maintien du niveau d'ingestion et la sélection d'une herbe plus digestible que l'herbe offerte, malgré la diminution de la biomasse produite par hectare, de la teneur en MAT et de la digestibilité de l'herbe offerte. La baisse de productivité 
Tableau VIII. Bilan annuel de pâturage sur la surface totale ou de prairies permanentes pâturée (3 ans).

\begin{tabular}{lccc}
\hline Traitement & Bas & Moyen & Haut \\
\hline $\begin{array}{l}\text { Surface maximale pâturée (ha total) } \\
\text { Jours de pâturage }\end{array} \quad 10,5$ & 8,8 & 7,2 \\
$\quad$ / ha total & 332 & 394 & 481 \\
$\quad$ ha P. permanentes & 456 & 550 & 689 \\
Lait $4 \%$ & & & \\
$\quad \mathrm{~kg} /$ ha total & 7518 & 8621 & 10608 \\
$\mathrm{~kg} /$ ha P. permanentes & 10709 & 12613 & 16050 \\
Récolte (t MS/ha total) & 2,7 & 3,2 & 3,4 \\
Concentré consommé (kg MS/ha total) & 869 & 1015 & 1306 \\
Fourrage consommé (kg MS/ha total) & 496 & 580 & 729 \\
Lait $4 \%$ autonome produit (kg/ha total) & 4810 & 5480 & 6510 \\
\hline
\end{tabular}

par hectare s'est alors avérée proportionnelle à la réduction de chargement induit par la réduction de l'intrant fertilisation azotée.

\subsection{Effet combiné de la fertilisation azotée et du chargement sur les conditions de pâturage}

Les travaux publiés sur la fertilisation azotée des prairies, et synthétisés dans divers ouvrages récents $[18,24,43,52]$, ont tous confirmés l'effet positif et décroissant de l'apport d'azote sur la production primaire des prairies. Les résultats obtenus au Pin au Haras sur prairies permanentes pâturées confortent cette loi générale : le rendement marginal de l'azote a été de $25 \mathrm{~kg}$ MS entre 0 et $100 \mathrm{~kg}$ d'azote et de $10 \mathrm{~kg}$ MS entre 100 et $320 \mathrm{~kg}$ d'azote. De même, les effets observés sur la composition chimique de l'herbe des prairies permanentes sont en accord avec ceux rapportés par d'autres études conduites sur prairies de graminées fauchées $[14,15,38,45,52]$. La diminution de la digestibilité consécutive à la réduction de la fertilisation azotée et estimée à partir de la digestibilité pepsine-cellulase ( $-0,02$ à $-0,05$ selon les cycles $)$, a été un peu plus importante que celle décrite classiquement [15, 38, 42].

Avec la réduction de la fertilisation azotée, la croissance de la plante et sa teneur en MAT deviennent plus dépendantes de la fourniture d'azote par le sol, elle même très variable selon les conditions agroclimatiques du milieu. Dans le milieu très favorable du Pin au Haras, en l'absence de toute fertilisation azotée, la prairie permanente a néanmoins produit $8,5 \mathrm{t}$ de $\mathrm{MS}$ d'une herbe dont la teneur en MAT et la valeur UFL sont restées, en moyenne annuelle, supérieures à $150 \mathrm{~g}$ et $0,83 \mathrm{UFL} / \mathrm{kg}$ MS.

Sur prairies temporaires, la présence du trèfle blanc a profondément modifié l'effet intrinsèque de la réduction de la fertilisation azotée sur la composition chimique de l'herbe des traitements Moyen et Bas. En accord avec les résultats de Simon [48], l'absence de fertilisation azotée a permis au trèfle blanc de se développer 
tandis qu'il a pratiquement disparu sur les prairies du traitement Haut. Ainsi, la teneur en MAT élevée et la grande stabilité de la digestibilité de la MO du trèfle blanc avec l'âge de la plante et la saison $[22,40]$ ont permis d'offrir aux animaux une herbe dont les caractéristiques ont été trés voisines voire supérieures à celles des prairies fertilisées.

En moyenne, grâce à une conduite du pâturage adaptée, la diminution de la production de MS/ha des prairies a été compensée par l'élargissement des surfaces pâturées. Ceci a permis d'offrir aux animaux des quantités d'herbe voisines (21 à $22 \mathrm{~kg} \mathrm{MS} / \mathrm{vache} / \mathrm{j}$ ). À ce niveau de quantités allouées, les conséquences des écarts observés au printemps, en faveur du traitement Moyen, sont modérées [41]. En revanche, la structure du couvert végétal, et notamment sa hauteur, ont été modifiées par la réduction de fertilisation azotée. En combinant hauteur et surface offertes, la mise à disposition de l'herbe a donc été différente entre traitements pour des quantités offertes similaires.

\subsection{Effet combiné de la fertilisation azotée et du chargement sur les performances zootechniques}

\subsubsection{Performances moyennes individuelles}

Au cours des 5 années d'expérience, la réduction ou la suppression de la fertilisation azotée n'ont pas altéré les performances individuelles journalières. Aucune tendance notable caractérisant une évolution négative de l'effet cumulé des traitements sur 5 années n'a pu être mis en évidence. Les travaux réalisés en Europe de l'Ouest ces dernières années confirment majoritairement la possibilité de produire autant de lait par vache et par jour sur prairies moins fertilisées à condition de modifier simultanément le chargement (tableau IX). Mais les niveaux de fertilisation azotée comparés sont générallement plus élevés. Ainsi, Lantinga et al. [34], aux Pays-Bas, ont obtenu des productions laitières identiques $(22,6 \mathrm{~kg}$ lait $4 \%)$ en réduisant la fertilisation de 520 à $320 \mathrm{~kg}$ $\mathrm{N} / \mathrm{ha} / \mathrm{an}$ et le chargement de 4,5 à 3,8 vache/ha. Deenen [8] rapporte les mêmes conclusions en pâturage continu suite à une expérience répétée durant 3 ans et impliquant 4 niveaux de fertilisation (250 à $700 \mathrm{~kg} \mathrm{~N} / \mathrm{ha}$ ). Des travaux conduits en France plus récemment [17, 23] mentionnent également le maintien des performances des vaches laitières.

Avec la réduction de la fertilisation azotée des prairies, à même niveau d'apport de concentré, les performances zootechniques peuvent être maintenues si les animaux sont capables de satisfaire leurs besoins en énergie et en azote grâce à un élargissement des surfaces en rapport avec la quantité et qualité de l'herbe produite.

À mêmes quantités d'herbe offertes, les quantités d'herbe ingérées, calculées à partir des mesures réalisées sur la prairie, n'ont pas différé lors des 3 dernières années d'expérience. En moyenne sur les cinq printemps, elles ont été un peu plus faibles chez les animaux du lot Bas. Le taux d'utilisation de l'herbe, défini comme le rapport entre les quantités d'herbe ingérées et d'herbe offerte (M. Journet, comm pers) a peu varié entre traitements et est resté inférieur à $85 \%$. La teneur en MAT de l'herbe est restée élevée, même sur les prairies permanentes non fertilisées. De plus, le concentré riche en tourteaux (54\%) et la présence de trèfle blanc sur prairies temporaires ont permis d'accroître la teneur en MAT de la ration. Les teneurs en urée du lait, supérieure à $25 \mathrm{mg} / 100 \mathrm{~mL}$ même en fin de parcelle, corroborent ces niveaux élevés d'apports azotés de la ration ([51]; Vérité et al., non publié). La digestibilité de l'herbe ingérée (dHI), supérieure à 0,77 , a été plus élevée que celle de l'herbe offerte (dHO). Les animaux des 
Tableau IX. Effet de la réduction de la fertilisation et du chargement sur la production laitière individuelle et par hectare. Synthèse bibliographique sur prairies de graminées.

\begin{tabular}{|c|c|c|c|c|c|c|c|}
\hline $\begin{array}{l}\text { Auteurs, référence } \\
\text { Durée exp. }\end{array}$ & $\begin{array}{c}\text { Fert } \\
(\mathrm{kg} \mathrm{N} / \mathrm{ha})\end{array}$ & $\begin{array}{l}\text { Chargt } \\
\text { (vl/ha) }\end{array}$ & JP/ha & $\begin{array}{l}\text { Lait / j } \\
(\mathrm{kg})\end{array}$ & $\begin{array}{l}\text { Lait / ha } \\
\text { (kg) }\end{array}$ & $\begin{array}{l}\text { JP / ha par } \\
\text { kg d'N (1) }\end{array}$ & $\begin{array}{l}\text { Lait / ha par } \\
\text { kg N (1) }\end{array}$ \\
\hline \multirow{4}{*}{$\begin{array}{l}\text { Cowan et Stobbs, [6] } \\
165 \mathrm{j}\end{array}$} & ] 100 & 1,90 & 313 & 10,0 & 3076 & \multirow[t]{2}{*}{0,99} & \multirow[t]{2}{*}{8,0} \\
\hline & 0 & 1,30 & 214 & 10,3 & 2272 & & \\
\hline & 100 & 2,50 & 412 & 8,9 & 3698 & \multirow[t]{2}{*}{1,48} & \multirow[t]{2}{*}{10,3} \\
\hline & 0 & 1,60 & 264 & 9,9 & 2673 & & \\
\hline \multirow{2}{*}{$\begin{array}{l}\text { Bienfait et al., [5] } \\
\quad 140 \mathrm{j}\end{array}$} & 180 & 4,00 & 560 & 15,6 & 8727 & \multirow[t]{2}{*}{1,17} & \multirow[t]{2}{*}{20,0} \\
\hline & 60 & 3,00 & 420 & 15,1 & 6326 & & \\
\hline \multirow{3}{*}{$\begin{array}{l}\text { Gately et al., [20] } \\
\quad 210 \mathrm{j}\end{array}$} & 400 & 3,11 & 654 & 10,3 & 6769 & \multirow{3}{*}{$\begin{array}{l}0,67 \\
2,22\end{array}$} & \multirow{3}{*}{$\begin{array}{c}7,4 \\
24,0\end{array}$} \\
\hline & 100 & 2,52 & 530 & 10,2 & 5394 & & \\
\hline & 50 & 2,00 & 419 & 10,0 & 4194 & & \\
\hline \multirow{4}{*}{$\begin{array}{l}\text { Davison et al., [7] } \\
\qquad 260 \mathrm{j}\end{array}$} & 400 & 3,00 & 818 & 10,3 & 8461 & \multirow[t]{2}{*}{1,31} & \multirow[t]{2}{*}{13,8} \\
\hline & 200 & 2,00 & 556 & 10,3 & 5707 & & \\
\hline & 400 & 3,50 & 902 & 10,2 & 9146 & \multirow[t]{2}{*}{1,29} & \multirow[t]{2}{*}{13,7} \\
\hline & 200 & 2,50 & 644 & 10,0 & 6410 & & \\
\hline \multirow{2}{*}{$\begin{array}{l}\text { Lantinga et al., [34] } \\
\qquad 175 \mathrm{j}\end{array}$} & 520 & 4,47 & 782 & 22,7 & 17774 & \multirow[t]{2}{*}{0,61} & \multirow[t]{2}{*}{14,6} \\
\hline & 320 & 3,77 & 660 & 22,5 & 14859 & & \\
\hline \multirow{2}{*}{$\begin{array}{l}\text { Fiorelli, [17] } \\
\qquad 144 \mathrm{j}\end{array}$} & 190 & 2,38 & 343 & 20,5 & 7327 & \multirow[t]{2}{*}{0,44} & \multirow[t]{2}{*}{8,0} \\
\hline & 66 & 2,00 & 288 & 20,9 & 6334 & & \\
\hline \multirow{4}{*}{$\begin{array}{l}\text { Deenen [8] } \\
\qquad 170 \mathrm{j} \text { - Pât continu }\end{array}$} & 700 & 4,05 & 690 & 22,9 & 15826 & \multirow{4}{*}{$\begin{array}{l}0,36 \\
0,50 \\
0,55\end{array}$} & \multirow{4}{*}{$\begin{array}{c}9,2 \\
12,4 \\
16,3\end{array}$} \\
\hline & u 550 & 4,00 & 680 & 22,7 & 15438 & & \\
\hline & 400 & 3,60 & 612 & 23,1 & 14149 & & \\
\hline & 250 & 3,11 & 529 & 22,1 & 11705 & & \\
\hline \multirow{2}{*}{$\begin{array}{l}\text { Deenen [8] } \\
\qquad 180 \mathrm{j} \text { - Pât tournant }\end{array}$} & 550 & 5,13 & 923 & 20,7 & 19161 & \multirow[t]{2}{*}{0,79} & \multirow[t]{2}{*}{14,1} \\
\hline & nt 250 & 3,80 & 685 & 21,8 & 14934 & & \\
\hline Delaby et al., [10] & 120 & 5,95 & 333 & 27,2 & 9060 & 1,80 & 48,5 \\
\hline $56 \mathrm{j}-$ Essai 1 & 40 & 3,37 & 189 & 27,4 & 5180 & & \\
\hline Delaby et al., [11] & 120 & 5,25 & 294 & 25,7 & 7555 & 0,84 & 28,9 \\
\hline $56 \mathrm{j}-$ Essai 2 & 40 & 4,05 & 227 & 23,1 & 5244 & & \\
\hline Dufrasne et al., [16] & 225 & 5,0 & 640 & 12,5 & $8782(*)$ & 0,70 & $5,1(*)$ \\
\hline 140 j - Pât continu & 110 & 4,0 & 560 & 14,6 & 8193 & & \\
\hline Dufrasne et al., [16] & 225 & 5,0 & 700 & 13,7 & 9584 & 1,22 & 12,8 \\
\hline $140 \mathrm{j}$ - Pât tournant & nt 110 & 4,0 & 560 & 14,5 & 8114 & & \\
\hline Hardy et al., [23] & 180 & 4,25 & 404 & 21,3 & 8715 & 1,11 & 23,5 \\
\hline $95 \mathrm{j}$ & 90 & 3,25 & 304 & 21,4 & 6600 & & \\
\hline
\end{tabular}

(1) Calculs réalisés par rapport au niveau de fertilisation le plus faible de chaque expérience. *Résultat incertain compte tenu des données disponibles dans la publication (durée de l'essai variable selon les traitements). 
traitements Moyen et Bas sont parvenus à compenser partiellement l'effet négatif de la réduction de fertilisation sur la digestibilité de l'herbe offerte.

Les caractéristiques du couvert végétal (biomasse/ha, hauteur, \% MAT, dHO...) n'ont pas, en moyenne, constitué un facteur limitant de l'ingestion pour les animaux des traitements Moyen et Bas. L'application d'un chargement différent, mais adapté aux différentes situations induites par le niveau de fertilisation azotée, a ainsi permis globalement des niveaux d'apports nutritifs et de production laitière identiques.

\subsubsection{Variabilité inter-annuelle des performances individuelles}

Néanmoins, lors de deux printemps en 1990 et 1994, la production de matières protéiques et de lait $4 \%$ a été plus faible chez les vaches du traitement Bas (figure 2). La production laitière maximale enregistrée 3 à $4 \mathrm{j}$ après l'entrée sur la parcelle [28], alors que les animaux ont à leur disposition des quantités d'herbe très élevées, a également été inférieure sur le traitement B. Dans d'autres conditions expérimentales, où la réduction de la fertilisation azotée conduit à des teneurs en MAT très faible du RGA (100 à $120 \mathrm{~g} / \mathrm{kg}$ MS), Delaby et al. [10, 11] ont montré qu'il n'était pas possible de maintenir les performances individuelles sauf à accroître fortement les quantités d'herbe offertes aux animaux. Delagarde et al. [13] ont confirmé cet effet en observant une baisse importante des quantités d'herbe ingérées sur prairies peu fertilisées malgré des quantités d'herbe offertes identiques entre traitements.

En fait, au cours des printemps 1990 et 1994, la hauteur de l'herbe à l'entrée $(<10 \mathrm{~cm})$ et le volume d'herbe offert ont été minimum ; les animaux ont été contraints de consommer une herbe plus rase. La structure de l'herbe des prairies du traitement Bas (hauteur, biomasse/ha) au travers la préhensibilité de l'herbe offerte a pu constitué un frein à l'ingestion [41] indépendamment de la quantité offerte. L'effet significatif du niveau de fertilisation sur les critères d'ingestion calculés (tableau $V$ ) est essentiellement dû aux écarts plus importants observés au printemps de ces 2 années (1990 et 1994). Mais, en l'absence d'interactions année $\times$ traitement significatives, la méthode de calcul des quantités ingérées (QHI et QTI) n'a pas permis de confirmer cette chute d'ingestion.

\subsubsection{Performances moyennes par hectare}

Sur l'ensemble du système de pâturage, la productivité des prairies du traitement Haut est voisine de celle rapportée par Hoden et al. [28] à même niveau de fertilisation azotée, mais a été obtenue avec des apports de concentré par hectare plus importants. Exprimée en lait $4 \%$ autonome [25], la production par hectare des prairies des traitements $\mathrm{M}$ et $\mathrm{B}$ a représenté 84 et $74 \%$ de celle du traitement Haut.

Au pâturage, la diminution de la fertilisation azotée entraîne inexorablement une réduction de la production laitière par hectare. Si les performances individuelles journalières sont maintenues, la réduction des performances par hectare est alors directement liée à la réduction du nombre de journées de pâturage réalisé par hectare. À partir des travaux recensés au tableau IX et pour lesquels la fertilisation azotée a été de $450 \mathrm{~kg} / \mathrm{ha} / \mathrm{an}$ maximum, nous avons calculé la relation entre le nombre de JP/ha réalisées et le niveau de fertilisation appliqué (Fert en $\mathrm{kg} \mathrm{N} / \mathrm{ha} / \mathrm{an}$ ) :

$$
\begin{gathered}
\mathrm{JP} / \mathrm{ha}=321+0,87( \pm 0,12) \\
\times \text { Fert }+ \text { effet Auteur } \\
\left(n=27-\text { Syx }=51-\mathrm{R}^{2}=0,96\right)[1]
\end{gathered}
$$


et la relation entre la production de lait/ha et la fertilisation. Cette dernière relation est très dépendante du potentiel de production des animaux utilisés dans chacun des essais. La quantité de lait journalière produite ( $\mathrm{PL}$ en $\mathrm{kg} / \mathrm{vl} / \mathrm{j})$ a donc été introduite dans le modèle selon un terme multiplicatif du niveau de fertilisation.

$$
\begin{gathered}
\text { Lait/ha }=5078+0,89( \pm 0,11) \\
\times \text { PL } \times \text { Fert }+ \text { effet Auteur } \\
\left(n=27-\text { Syx }=646-\mathrm{R}^{2}=0,97\right)
\end{gathered}
$$

En dessous d'une fertilisation annuelle de $450 \mathrm{~kg} / \mathrm{ha}$, la relation est linéaire et chaque $\mathrm{kg}$ d'azote apporté en moins à la prairie conduit à une réduction de 0,87 journée de pâturage. Avec une production laitière journalière moyenne entre essais de $15,8 \mathrm{~kg}$ de lait, la réduction de fertilisation entraîne une baisse de productivité des prairies de $14,1 \mathrm{~kg}$ de lait/ha par $\mathrm{kg}$ d'azote. Cette réponse moyenne est en accord avec les résultats rapportés par Journet et Demarquilly [30], mais varie beaucoup avec le potentiel de production des animaux.

Sur les prairies permanentes, la production laitière sans fertilisation azotée a atteint $10710 \mathrm{~kg}$ lait $4 \%$ /ha pour 456 journées de pâturage par hectare (tableau VIII). La réponse marginale en terme de JP/ha a été de 0,63 entre 100 et $320 \mathrm{~kg} \mathrm{~N} / \mathrm{ha}$ et a atteint 0,94 entre 0 et $100 \mathrm{~kg} \mathrm{~N} / \mathrm{ha}$. Ces valeurs encadrent la valeur de la pente de l'équation [1] décrite ci-dessus. La réponse de production laitière a atteint sur ces parcelles respectivement 15,6 et $19,0 \mathrm{~kg}$ de lait/ha par $\mathrm{kg} \mathrm{d}$ 'N. Si l'on admet une production moyenne journalière de $23,2 \mathrm{~kg}$ sur prairies permanentes, la réponse attendue serait de $20,6 \mathrm{~kg}$ de lait selon le coefficient de l'équation (2).

\section{CONCLUSION}

Cette synthèse expérimentale conforte l'idée avancée par Gately et al. [20], qui suggèrent qu'à chaque niveau de fertili- sation azotée correspond un niveau de chargement adapté qui permet les mêmes performances individuelles. Toute la difficulté réside dans la définition de ce chargement optimum [7, 30], qui avec la réduction de la fertilisation azotée devient plus, voire très, dépendant des potentialités agronomiques locales et annuelles. En l'absence de fertilisation azotée, la variabilité entre années risque d'être accrue dans la mesure où les apports d'azote exogéne via la fertilisation n'atténuent plus les variations de fourniture d'azote par le sol via la minéralisation.

\section{REMERCIEMENTS}

Les auteurs tiennent à remercier M. Journet et $\mathrm{A}$. Hoden qui ont initié ce programme de recherches et l'ensemble du personnel du domaine Inra du Pin au Haras pour sa contribution assidue et efficace à l'ensemble des prélèvements et mesures réalisés au cours des cinq années.

Cette expérience pluriannuelle a été réalisée lors d'une action incitative programmée « Recherche de systèmes extensifiés de production bovine pour une utilisation accrue des surfaces agricoles en herbe, la réduction des intrants et la sauvegarde de l'environnement », décidée et financée par la direction de l'Inra dans le cadre d'une recherche pluridisciplinaire.

\section{RÉFÉRENCES}

[1] Agabriel J., Giraud J.M., Petit M., Détermination et utilisation de la note d'état d'engraissement en élevage allaitant, Bull. Tech. C.R.Z.V., Theix, Inra 66 (1986), 43-50.

12] Aufrère J., Demarquilly C., Predicting organic matter digestibility of forage by two pepsin-cellulase methods, XVI Congrès International des Herbages 4-11 Octobre (A.F.P.F., ed.), Nice, 1989, pp. 877-878.

[3] Beranger C., La simplification du pâturage ? Introduction générale, Fourrages 111 (1987) 231-238.

[4] Beranger C., Micol D., Utilisation de l'herbe par les bovins au pâturage. Importance du chargement et du mode d'exploitation, Fourrages 85 (1981) 73-93. 
15] Bienfait J.M., Van Eenaeme C., Limbourg P., Comparaison de quatre systèmes de pâturage différant par les chargements, les doses de complément énergétique et les niveaux de fumure azotée en fonction de la productivité de l'herbe et de la production laitière sur les pâturages d'Ardennes belges, Fourrages 74 (1978) 19-28.

[6] Cowan R.T., Stobbs T.H., Effects of nitrogen fertilizer applied in autumn and winter on milk production from a tropical grasslegume pasture grazed at four stocking rates, Aust. J. Exp. Agric. 16 (1976) 829-837.

[7] Davison T.M., Cowan R.T., Shepherd R.K., Milk production from cows grazing on tropical pastures. 2. Effects of stocking rate and level of nitrogen fertilizer on milk yield and pasture-milk yield relationships, Aust. J. Exp. Agric. 25 (1985) 515-523.

[8] Deenen P.J.A.G., Nitrogen use efficiency in intensive grassland farming, Thèse, Department of Agronomy, Wageningen, Pays-Bas, 1994.

[9] Delaby L., Peyraud JL., Hoden A., Muller A., Peccatte J.R., Réduction de la fertilisation azotée et production laitière au pâturage, in : L'extensification fourragère (A.F.P.F., éd.) Paris, France, 1992, 64-65.

[10] Delaby L., Peyraud J.L., Vérité R., Marquis B., Effect of protein content in the concentrate and level of nitrogen fertilization on the performance of dairy cows in pasture, Ann. Zootech. 45 (1996) 327-341.

[11] Delaby L., Bouttier A., Peyraud J.L., Peccatte J.R., Effet d'une réduction simultanée de la fertilisation azotée et du chargement sur les performances des vaches laitières au pâturage, troisième Renc. Rech. Ruminants, 4-5 Décembre, Paris-La Villette, 1996, 98 p.

[12] Delaby L., Decau M.L., Peyraud J.L., Accarie P., AzoPât : Une description quantifiée des flux annuels d'azote en prairie pâturée par les vaches laitières. 1. Les flux associés à l'animal, Fourrages 151 (1997) 297-311.

[13] Delagarde R., Peyraud J.L., Delaby L., The effect of nitrogen fertilization level and protein supplementation on herbage intake, feeding behaviour and digestion in grazing dairy cows, Anim. Feed. Sci. Technol. 66 (1997) 165-180.

[14] Demarquilly C., Influence de la fertilisation azotée sur la valeur alimentaire des fourrages verts, Ann. Zootech. 19 (1970) 423-437.

[15] Demarquilly C., Fertilisation et qualité du fourrage, Fourrages 69 (1977) 61-84.

[16] Dufrasne I., Gielen M., Limbourg P., Korsak N., Istasse L., Effets d'une augmentation de la fumure azotée ou de la distribution supplémentaire de concentré sur les performances et les teneurs en urée plasmatique de vaches laitières soumises au pâturage continu ou en rotation, Ann. Zootech. 45 (1996) 135-150.

[17] Fiorelli J.L., Extensification du pâturage continu des vaches laitières, in : L'extensification fourragère (A.F.P.F., éd.), Paris, France, 1992, 68-69.

[18] Frame J., Improved Grassland Management, Farming Press Books, Ipswich, RoyaumeUni, 1992, pp. 351.

[19] François E., Pithan K., Bathiaux-Thill N., (eds.) Nitrogen cycling and leaching in cool and weet regions of Europe, CEC, Gembloux, Belgique, 1992, pp. 149, COST 814.

[20] Gately T.F., O'Keffe W.F., Connoly J., Review of nitrogen and stocking rate experiments for milk production in Ireland, Ir. J. Agric. Res. 23 (1984) 1 1-26.

[21] Germon J.C., (ed.) Management systems to reduce impact of nitrates, Elsevier Science Publishers, Londres, Royaume-Uni, 1989, pp. 274.

[22] Giovanni R., Valeur alimentaire des associations graminées/trèfle blanc, Inra Prod. Anim. 1 (1988) 193-200.

[23] Hardy A., Rivière F., Gastebled A., Influence de la quantité d'herbe offerte ou de la réduction de la fertilisation azotée sur les performances des vaches laitières au pâturage, troisième Renc. Rech. Ruminants, 4-5 Décembre, Paris-La Villette, 1996, 85-88.

124] Hnatyszyn M., Guais A., Les fourrages et l'éleveur, Lavoisier, Tech. Doc., Paris, France, 1988, pp. 440.

[25] Hoden A., Micol D., Liénard G., Muller A., Peyraud J.L., Interprétation des essais de pâturage avec des bovins : terminologie, modes de calcul, bilans annuels, Bull. Tech. CRZV Theix Inra 63 (1986) 31-42.

[26] Hoden A., Muller A., Journet M., Faverdin P., Pâturage pour vaches laitières. 1. Comparaison des systèmes de pâturage rationné et tournant simplifié en zone normande, Bull. Tech. CRZV Theix Inra 64 (1986) 25-35.

[27] Hoden A., Fiorelli J.L., Jeannin B., Huguet L., Muller A., Weiss P., Pâturage simplifié pour vaches laitières : synthèse de résultats expérimentaux, Fourrages 111 (1987) 239-257.

[28] Hoden A., Peyraud J.L., Muller A., Delaby L., Faverdin P., Simplified rotational grazing management of dairy cows: effects of rates of stocking and concentrate, J. Agric. Sci, Camb. 116 (1991) 417-428.

[29] Inra, Alimentation des ruminants : Révision des systèmes et des tables de l'Inra, Bull. Tech. CRZV Theix Inra 70, 1987, pp. 222.

[30| Journet M., Demarquilly C., Grazing, in: Feeding strategy for the high yielding cow (Broster W.H., Swan H., eds.), Granada Publishing Ltd., Londres, 1979, 295-321. 
[31] Kanneganti V.R., Kaffka S.R., Forage availability from a temperate pasture managed with intensive rotational grazing, Grass For. Sci. 50 (1995) 50-62.

[32] Laissus R., Comparaison des résultats d'essais de fourrages récoltés en fauche ou en pâture, Fourrages 43 (1970) 45-56.

[33] Lantinga E.A., Simulation of herbage production and herbage intake during a rotational grazing period: An evaluation of Linehan's formula, Neth. J. Agric. Sci. 33 ( 1985 ) 385-403.

[34] Lantinga E.A., t'Mannetje L., Schlepers H., Relations between nitrogen fertilization, herbage production and milk yield, XVI Congrès international des herbages, 4-11 octobre (A.F.P.F., éd.), Nice, France, 1989, pp. 1094-1095.

[35] Lemaire G., Salette J., Laissus R., Analyse de la croissance d'une prairie naturelle normande au printemps. I. La production et sa variabilité, Fourrages 91 (1982) 3-16.

[36] Liénard G., Cordonnier P., Boutonnet J.P., Exploitations et systèmes de production d'herbivores. Importance, évolution, questions, Inra Prod. Anim. 5 (1992) 59-85.

[37] Michel M.C., Analyse quantitative de quelques substances azotées et glucidiques en milieu biologique. Essai de rationalisation, Thèse de doctorat, université de Clermont-II, Clermont-Ferrand, France, 1971.

[38] Minson D.J., Forage in Ruminant Nutrition, Academic Press, Inc., San Diego, CA, ÉtatsUnis, 1990, pp. 483.

[39] Peyraud J.L., Delaby L., Effet de la réduction de la fertilisation azotée sur la qualité de l'herbe sélectionnée par les vaches laitières. In : L'extensification fourragère, (A.F.P.F., ed.) Paris, France, (1992) 128-129.

[40] Peyraud J.L., Comparaison de la digestion du trèfle blanc et des graminées prairiales chez la vache laitière, Fourrages 135 (1993) 465-473.

[41] Peyraud J.L., Comeron E.A., Wade M.H., Lemaire G., The effect of daily herbage allowance, herbage mass and animal factors upon herbage intake by grazing dairy cows, Ann. Zootech. 45 (1996) 201-217.

[42] Peyraud J.L., Astigarraga L., Review of the effect of nitrogen fertilization on the chemical composition, intake, digestion and nutritive value of fresh herbage: consequences on animal nutrition and $\mathrm{N}$ balance, Anim. Feed Sci. Technol. (1998) in press.
[431 Prins W.H., Postmus J., Reker A.M., Ruiter B., Nitrogen use on grassland in spring, in the Netherlands and elsewhere in Europe: Temperature sum, stage of growth, rate and source of nitrogen, Neth. Fertilizer Techn. Bull. 17 (1988) 1-55.

[44) Reid D., The effect of long-term application of a wide range of nitrogen rates on the yields from perennial ryegrass swards with and without white clover, J. Agric. Sci. Camb. 79 (1972) 291-301.

[45] Reid D., Strachan N.H., The effects of a wide range of nitrogen rates on some chemical constituents of the herbage from perennial ryegrass with and without white clover, J. Agric. Sci. Camb. 83 (1974) 393-401.

[46] SAS Institute, Inc., SAS/Stat Guide for Personal Computer, Version 6, Cary, N.C., U.S.A. (1987).

[47] Salette J., Huché L., Diagnostic de l'état de nutrition minérale d'une prairie par l'analyse du végétal : principes, mise en æuvre et exemples, Fourrages 125 (199!) 3-18.

[48] Simon J.C., Azote et équilibre de l'association ray-grass anglais-trèfle blanc, XVI Congrès International des Herbages,4-11 Octobre (A.F.P.F., ed.), Nice, France (1989) 471-472.

[49] Simon J.C., Peyraud J.L., Decau M.L., Delaby L., Vertès F., Delagarde R., Gestion de l'azote dans les systèmes prairiaux pâturés. In Maitrise de l'azote dans les agrosystèmes (Lemaine S, Nicolerdot B, eds), Colloque Inra, 19-20 Novembre 1996, Reims, pp. 201-216.

[50] Urban B., Caudal J.P., Herbomètre automatisé, In Les journées de la mesure. Electronique, informatique, automatique (Inra dépt. Informatique, ed.) 3-4 Octobre, Port Leucate, II, (1990), 57-59.

[51] Vérité R., Rétif S., Faverdin P., L’urée du lait comme index de la qualité de la nutrition azotée et de l'excrétion d'azote chez la vache laitière en alimentation hivernale, deuxième Renc. Rech. Ruminants, 13-14 Décembre, Paris-La Villette, p. 365 (1995)

[52] Whitehead D.C., Grassland Nitrogen, CAB International, Wallingford, United Kingdom (1995), pp. 397.

[53] Wilman D., Wright P.T., The proportions of cell content, nitrogen, nitrate-nitrogen and water-soluble carbohydrate in three grasses in the early stages of regrowth after defoliation with and without applied nitrogen, J. Agric. Sci. Camb. 91 (1978) 381-394. 